\title{
NAMBA FORCING, WEAK APPROXIMATION, AND GUESSING
}

\author{
SEAN COX AND JOHN KRUEGER
}

\begin{abstract}
We prove a variation of Easton's lemma for strongly proper forcings, and use it to prove that, unlike the stronger principle IGMP, GMP together with $2^{\omega} \leq \omega_{2}$ is consistent with the existence of an $\omega_{1}$-distributive nowhere c.c.c. forcing poset of size $\omega_{1}$. We introduce the idea of a weakly guessing model, and prove that many of the strong consequences of the principle GMP follow from the existence of stationarily many weakly guessing models. Using Namba forcing, we construct a model in which there are stationarily many indestructibly weakly guessing models which have a bounded countable subset not covered by any countable set in the model.
\end{abstract}

Weiss [15] introduced the combinatorial principle ISP $(\kappa)$, which characterizes supercompactness in the case that $\kappa$ is inaccessible, but is also consistent for small values of $\kappa$ such as $\omega_{2}$. The principle ISP $\left(\omega_{2}\right)$ follows from PFA, and ISP $\left(\omega_{2}\right)$ implies some of the strong consequences of PFA, such as the failure of the square principle at all uncountable cardinals. Viale-Weiss [14] introduced the idea of an $\omega_{1}$-guessing model, and proved that ISP $\left(\omega_{2}\right)$ is equivalent to the existence of stationarily many $\omega_{1}$-guessing models in $P_{\omega_{2}}(H(\theta))$, for all cardinals $\theta \geq \omega_{2}$.

Viale [13] proved that the singular cardinal hypothesis $(\mathrm{SCH})$ follows from the existence of stationarily many $\omega_{1}$-guessing models which are also internally unbounded, which means that any countable subset of the model is covered by a countable set in the model. This raises the question of whether $\operatorname{ISP}\left(\omega_{2}\right)$ alone implies $\mathrm{SCH}$. A closely related question of Viale [13, Remark 4.3] is whether it is consistent to have $\omega_{1}$-guessing models which are not internally unbounded. Much of the work in this paper was motivated by these two questions.

In this paper we introduce a weak form of $\omega_{1}$-guessing. Let $\kappa$ be a regular uncountable cardinal. A model $N$ of size $\omega_{1}$ with $\kappa \in N$ is said to be weakly $\kappa$ guessing if whenever $f: \sup (N \cap \kappa) \rightarrow O n$ is a function such that for cofinally many $\alpha<\sup (N \cap \kappa), f\lceil\alpha \in N$, then there is a function $g \in N$ with domain $\kappa$ such that $g\lceil\sup (N \cap \kappa)=f$. We say that $N$ is weakly guessing if $N$ is weakly $\kappa$-guessing for all regular uncountable cardinals $\kappa \in N$. We will show that the existence of stationarily many weakly guessing models suffices to prove most of the strong consequences of $\operatorname{ISP}\left(\omega_{2}\right)$, including the failure of square principles.

Date: September 2016; revised May 2018.

2010 Mathematics Subject Classification: 03E05, 03E35, 03E40, 03E65.

Key words and phrases. Weak $\omega_{1}$-appoximation property, weak $\omega_{1}$-guessing, wGMP, forcing axioms, Namba forcing.

The work on this paper began while the authors attended the "High and low forcing" workshop at the American Institute of Mathematics in January 2016. The first author was partially supported by the Simons Foundation grant 318467 and the VCU Presidential Research Quest Fund. The second author was partially supported by the National Science Foundation Grant No. DMS-1464859. 
By passing through the idea of a weakly $\omega_{1}$-guessing model, we solve an easy special case of the problem of Viale stated above, showing that the existence of $\omega_{1}$ guessing models implies the existence of $\omega_{1}$-guessing models $N$ such that $\sup (N \cap$ $O n$ ) has cofinality $\omega$, and in particular, $N$ is not internally unbounded. This result suggests a refined version of Viale's question: is every bounded countable subset of an $\omega_{1}$-guessing model covered by a countable set in the model? If the answer is yes, then ISP $\left(\omega_{2}\right)$ does indeed imply SCH. This problem remains open.

The main result of this paper is the consistency that there are stationarily many indestructibly weakly guessing models $N \in P_{\omega_{2}}\left(H\left(\aleph_{\omega+1}\right)\right)$ for which there is a countable subset of $N \cap \aleph_{\omega}$ which is not covered by any countable set in $N$. This result can be thought of as a first attempt towards proving that $\operatorname{ISP}\left(\omega_{2}\right)$ does not imply $\mathrm{SCH}$. The proof involves constructing a model in which there exists a diagonal form of Namba forcing which satisfies a weak version of the $\omega_{1}$-approximation property.

Main Theorem. It is consistent relative to the existence of a supercompact cardinal with infinitely many measurable cardinals above it that there exist stationarily many $N \in P_{\omega_{2}}\left(H\left(\aleph_{\omega+1}\right)\right)$ such that $N$ is indestructibly weakly guessing, has uniform cofinality $\omega_{1}$, and is not internally unbounded.

Before starting the main line of results concerning weakly guessing models and covering, we begin the paper by proving a variation of the classical Easton's lemma for strongly proper forcing: if $\mathbb{P}$ is strongly proper on a stationary set and $\mathbb{Q}$ is $\omega_{1}$-closed, then $\mathbb{P}$ forces that $\mathbb{Q}$ is $\omega_{1}$-distributive. As a corollary, we will show that a certain combinatorial principle known to follow from the existence of an indestructible version of an $\omega_{1}$-guessing model does not follow from the existence of $\omega_{1}$-guessing models.

We give a brief outline of the contents of the paper. Section 1 describes some background material which will be needed to understand the paper. Section 2 presents our strongly proper variation of Easton's lemma.

Section 3 defines weak approximation and weak guessing, and proves that many of the strong consequences of ISP $\left(\omega_{2}\right)$ follow from the existence of stationarily many weakly guessing models. Section 4 solves a problem of Viale [13 by showing the consistency that there are stationarily many $\omega_{1}$-guessing models which are not internally unbounded.

Section 5 shows that the method of Viale-Weiss 14 for applying forcing axioms to prove the existence of $\omega_{1}$-guessing models can be adapted to the case of weakly guessing models. Section 6 develops a version of Namba forcing which has the weak approximation property. Section 7 proves the main theorem of the paper, showing that it is consistent that there exist stationarily many indestructibly weakly guessing models which have a bounded countable subset not covered by any countable set in the model.

We would like to thank Thomas Gilton for carefully proofreading several drafts of this paper and making many useful suggestions.

\section{BACKGROUND}

We assume that the reader has a working knowledge of forcing, proper forcing, product forcing, the product lemma, finite step forcing iterations, and generalized 
stationarity. The reader should be familiar with trees of height $\omega_{1}$ and with the standard $\omega_{1}$-c.c. forcing poset for adding a specializing function to a tree with no uncountable chains.

For a regular uncountable cardinal $\kappa$, we say that a forcing poset is $\kappa$-closed if any descending sequence of conditions of length less than $\kappa$ has a lower bound. We say that a forcing poset is $\kappa$-distributive if it does not add new sets of ordinals of size less than $\kappa$.

Definition 1.1. Let $N$ be a set with $|N|=\omega_{1}$ and $\omega_{1} \subseteq N$. We say that $N$ has bounded uniform cofinality $\omega_{1}$ if for all $\alpha \in N$, if $N \models \operatorname{cf}(\alpha)>\omega$ then $\operatorname{cf}(\sup (N \cap \alpha))=\omega_{1}$. If in addition we have that $\operatorname{cf}(\sup (N \cap O n))=\omega_{1}$, then we say that $N$ has uniform cofinality $\omega_{1}$.

Note that if $N \prec H(\theta)$ for some cardinal $\theta \geq \omega_{2}$, then $N$ has bounded uniform cofinality $\omega_{1}$ iff for every regular uncountable cardinal $\lambda \in N, \operatorname{cf}(\sup (N \cap \lambda))=\omega_{1}$.

A set of ordinals $a$ is said to be countably closed if every limit point of $a$ with countable cofinality is in $a$.

The following fact is well-known.

Lemma 1.2. Let $N$ be a set with $|N|=\omega_{1}$ and $\omega_{1} \subseteq N$. Suppose that $N$ is an elementary substructure of $H(\theta)$ for some cardinal $\theta \geq \omega_{2}$ and $N$ has bounded uniform cofinality $\omega_{1}$. Then for any ordinal $\alpha \in N$ with uncountable cofinality, $N \cap \alpha$ is countably closed.

Proof. Let $\beta$ be a limit point of $N \cap \alpha$ with countable cofinality, and we will show that $\beta \in N \cap \alpha$. Since $\operatorname{cf}(\sup (N \cap \alpha))=\omega_{1}, \beta<\sup (N \cap \alpha)$. Let $\xi:=\min ((N \cap$ $\alpha) \backslash \beta)$.

Suppose for a contradiction that $\beta \notin N$. Then $\beta<\xi$ and $N \cap \xi=N \cap \beta$. Clearly $\operatorname{cf}(\xi)>\omega$, for otherwise by elementarity $N \cap \xi$ would be cofinal in $\xi$. Since $N$ has bounded uniform cofinality $\omega_{1}, \operatorname{cf}(\sup (N \cap \xi))>\omega$. But $\sup (N \cap \xi)=\beta$ and $\operatorname{cf}(\beta)=\omega$, which is a contradiction.

Definition 1.3. Let $N$ be an uncountable set with $\omega_{1} \subseteq N$. We say that $N$ is $\omega_{1}$-guessing if for any set of ordinals $d \subseteq N$ such that $\sup (d)<\sup (N \cap O n)$, if $d$ satisfies that for any countable set $b \in N, d \cap b \in N$, then there exists $d^{\prime} \in N$ such that $d=d^{\prime} \cap N$.

Definition 1.4. Let $W_{1}$ and $W_{2}$ be transitive sets or classes with $W_{1} \subseteq W_{2}$. We say that the pair $\left(W_{1}, W_{2}\right)$ has the $\omega_{1}$-approximation property if whenever $d \in W_{2}$ is a bounded subset of $W_{1} \cap O n$ and satisfies that $b \cap d \in W_{1}$ for any set $b \in W_{1}$ which is countable in $W_{1}$, then $d \in W_{1}$.

Lemma 1.5. Let $N$ be an elementary substructure of some transitive structure which satisfies ZFC - Powerset and correctly computes $\omega_{1}$. Then the following are equivalent:

(1) $N$ is $\omega_{1}$-guessing;

(2) the pair $\left(N_{0}, V\right)$ has the $\omega_{1}$-approximation property, where $N_{0}$ is the transitive collapse of $N$.

Proof. See the proof of [1, Lemma 1.10].

Definition 1.6. A forcing poset $\mathbb{P}$ is said to have the $\omega_{1}$-approximation property if $\mathbb{P}$ forces that $\left(V, V^{\mathbb{P}}\right)$ has the $\omega_{1}$-approximation property. 
For a set $M$ and a filter $G$ on a forcing poset $\mathbb{P}$, we say that $G$ is $M$-generic if for any dense subset $D$ of $\mathbb{P}$ which is a member of $M, G \cap M \cap D \neq \emptyset$.

Definition 1.7. Let $\mathbb{P}$ be a forcing poset and $N$ a set with $N \cap \mathbb{P} \neq \emptyset$. A condition $q$ is said to be strongly $(N, \mathbb{P})$-generic if for any dense subset $D$ of the forcing poset $N \cap \mathbb{P}, D$ is predense below $q$.

If $\mathbb{P}$ is understood from context, we say that $q$ is strongly $N$-generic if $q$ is strongly $(N, \mathbb{P})$-generic.

Under some non-triviality assumptions on $N$, it is easy to check that $q$ is strongly $(N, \mathbb{P})$-generic iff $q$ forces that $\dot{G}_{\mathbb{P}} \cap N$ is a $V$-generic filter on $N \cap \mathbb{P}$. For example, this is true if any conditions $s$ and $t$ in $N \cap \mathbb{P}$ which are compatible in $\mathbb{P}$ are also compatible in $N \cap \mathbb{P}$.

Definition 1.8. Let $\mathbb{P}$ be a forcing poset and $N$ a set. We say that $\mathbb{P}$ is strongly proper for $N$ if for all $p \in N \cap \mathbb{P}$, there is $q \leq p$ such that $q$ is strongly $(N, \mathbb{P})$-generic.

Definition 1.9. Let $\kappa$ be a regular uncountable cardinal. A forcing poset $\mathbb{P}$ is $\kappa$ strongly proper on a stationary set if for all sufficiently large cardinals $\chi \geq \kappa$ with $\mathbb{P} \in H(\chi)$, there are stationarily many $N \in P_{\kappa}(H(\chi))$ with $N \cap \kappa \in \kappa$ such that $\mathbb{P}$ is strongly proper for $N$. We say that $\mathbb{P}$ is $\kappa$-strongly proper if the stationary set just described contains a club.

A forcing poset $\mathbb{P}$ is strongly proper on a stationary set if $\mathbb{P}$ is $\omega_{1}$-strongly proper on a stationary set, and $\mathbb{P}$ is strongly proper if $\mathbb{P}$ is $\omega_{1}$-strongly proper. The ideas of strong genericity and strongly proper are due to Mitchell.

As discussed in [1, Section 2], letting $\lambda_{\mathbb{P}}$ be the first cardinal greater than or equal to $\kappa$ such that $\mathbb{P} \subseteq H\left(\lambda_{\mathbb{P}}\right), \mathbb{P}$ is $\kappa$-strongly proper on a stationary set iff there are stationarily many $N \in P_{\kappa}\left(H\left(\lambda_{\mathbb{P}}\right)\right)$ with $N \cap \kappa \in \kappa$ such that $\mathbb{P}$ is strongly proper for $N$.

The following well-known result is due to Mitchell; see [9, Lemma 6].

Theorem 1.10. Let $\kappa$ be a regular uncountable cardinal, and assume that $\mathbb{P}$ is $\kappa$-strongly proper on a stationary set. Then $\mathbb{P}$ forces that for any set of ordinals $X$, if $X \cap a \in V$ for any set $a \in V$ of size less than $\kappa$ in $V$, then $X \in V$.

In particular, if $\mathbb{P}$ is strongly proper on a stationary set, then $\mathbb{P}$ has the $\omega_{1}$ approximation property.

Weiss introduced principles $\operatorname{ITP}(\kappa)$ and $\operatorname{ISP}(\kappa)$ which give a combinatorial characterization of supercompactness in the case that $\kappa$ is inaccessible, but also make sense when $\kappa$ is a small cardinal such as $\omega_{2}$. We refer the reader to [15, Section 2] for the definitions. The principle $\operatorname{ISP}(\kappa)$ implies $\operatorname{ITP}(\kappa)$, and $\operatorname{ISP}\left(\omega_{2}\right)$ follows from PFA.

The following theorem was proved in [14, Section 3].

Theorem 1.11. The principle $\operatorname{ISP}\left(\omega_{2}\right)$ is equivalent to the statement that for all cardinals $\chi \geq \omega_{2}$, there are stationarily many $N \in P_{\omega_{2}}(H(\chi))$ such that $N$ is $\omega_{1}$-guessing.

For a cardinal $\lambda \geq \omega_{2}$, let $\operatorname{GMP}(\lambda)$ be the statement that there exist stationarily many sets in $P_{\omega_{2}}(H(\lambda))$ which are $\omega_{1}$-guessing. Let GMP be the statement that $\operatorname{GMP}(\lambda)$ holds for all cardinals $\lambda \geq \omega_{2}$. By Theorem 1.11, GMP is equivalent to $\operatorname{ISP}\left(\omega_{2}\right)$. 
For a cardinal $\lambda \geq \omega_{2}$, let IGMP $(\lambda)$ be the statement that there exist stationarily many sets in $P_{\omega_{2}}(H(\lambda))$ which are $\omega_{1}$-guessing in any generic extension by an $\omega_{1}$-preserving forcing poset. Let IGMP be the statement that $\operatorname{IGMP}(\lambda)$ holds for all cardinals $\lambda \geq \omega_{2}$. We refer the reader to [2] for more information about indestructible guessing models.

\section{A VARIATION OF EASTON'S LEMMA}

Before starting the main topic of the paper, we will prove in this section a strongly proper variation of Easton's lemma, together with a corollary which further distinguishes the principles GMP and IGMP.

By Todorčević's maximality principle we will mean the statement that any forcing poset which adds a new subset of $\omega_{1}$ whose proper initial segments are in the ground model collapses either $\omega_{1}$ or $\omega_{2}$. This statement was introduced by Todorčević [12] and shown to follow from some combinatorial assumptions about trees.

In [2, Theorem 3.9] we proved that the principle IGMP together with $2^{\omega} \leq \omega_{2}$ imply Todorčević's maximality principle. We will show below that IGMP cannot be weakened to GMP in this result. The proof will use a new variation of the classical Easton's lemma.

Theorem 2.1 (Easton's lemma). Suppose that $\kappa$ is a regular uncountable cardinal, $\mathbb{P}$ is $\kappa$-c.c., and $\mathbb{Q}$ is $\kappa$-closed. Then $\mathbb{P}$ forces that $\mathbb{Q}$ is $\kappa$-distributive.

In particular, if $\mathbb{P}$ is $\omega_{1}$-c.c. and $\mathbb{Q}$ is $\omega_{1}$-closed, then $\mathbb{P}$ forces that $\mathbb{Q}$ is $\omega_{1}$ distributive.

We introduce a variation of Easton's lemma in which the same conclusion follows from $\mathbb{P}$ being strongly proper on a stationary set in place of being $\omega_{1}$-c.c. We will use the next result which we proved in [2, Theorem 5.5].

Theorem 2.2. Suppose that $\mathbb{P}$ is strongly proper on a stationary set and $\mathbb{Q}$ is proper. Then $\mathbb{Q}$ forces that $\mathbb{P}$ is strongly proper on a stationary set.

Theorem 2.3 (Variation of Easton's lemma). Suppose that $\mathbb{P}$ is strongly proper on a stationary set and $\mathbb{Q}$ is $\omega_{1}$-closed. Then $\mathbb{P}$ forces that $\mathbb{Q}$ is $\omega_{1}$-distributive.

Proof. Let $G \times H$ be a generic filter on $\mathbb{P} \times \mathbb{Q}$. By the product lemma, we have that $V[G \times H]=V[G][H]=V[H][G]$, where $H$ is a $V[G]$-generic filter on $\mathbb{Q}$ and $G$ is a $V[H]$-generic filter on $\mathbb{P}$. So to show that $\mathbb{Q}$ is $\omega_{1}$-distributive in $V[G]$, it suffices to show that if $f: \omega \rightarrow O n$ is a function in $V[G \times H]$, then $f \in V[G]$. Fix a $\mathbb{P}$-name $\dot{f}$ in $V[H]$ such that $\dot{f}^{G}=f$.

Since $\mathbb{Q}$ is $\omega_{1}$-closed, it is proper. As $\mathbb{P}$ is strongly proper on a stationary set in $V$, it follows by Theorem 2.2 that $\mathbb{P}$ is strongly proper on a stationary set in $V[H]$.

Working in $V[H]$, fix a regular cardinal $\theta$ large enough so that $\mathbb{P}, \mathbb{Q}$, and $\dot{f}$ are in $H(\theta)$. Since $\mathbb{P}$ is strongly proper on a stationary set in $V[H]$, it follows that there are stationarily many $M \in P_{\omega_{1}}(H(\theta))$ with $M \prec(H(\theta), \in, \mathbb{P}, \dot{f})$ such that every condition in $M \cap \mathbb{P}$ has an extension which is strongly $(M, \mathbb{P})$-generic.

Applying the $V[H]$-genericity of $G$, an easy density argument shows that for some $M$ as described in the previous paragraph, $G$ contains a strongly $(M, \mathbb{P})$ generic condition. Since $G$ contains a strongly $(M, \mathbb{P})$-generic condition, it follows that $g_{M}:=G \cap M$ is a $V[H]$-generic filter on the forcing poset $M \cap \mathbb{P}$. 
Since $\dot{f} \in M$, by elementarity we have that for each $n<\omega$, the dense set $D_{n}$ of conditions in $\mathbb{P}$ which decide the value of $\dot{f}(n)$ is in $M$. Again by elementarity, it follows that $D_{n} \cap M$ is a dense subset of $M \cap \mathbb{P}$. Since $g_{M}$ is a $V[H]$-generic filter on $M \cap \mathbb{P}, g_{M} \cap D_{n} \neq \emptyset$. It easily follows that for all $n<\omega$ and $\alpha$,

$$
f(n)=\alpha \Longleftrightarrow \exists t \in g_{M}, t \Vdash_{\mathbb{P}}^{V[H]} \dot{f}(n)=\check{\alpha} .
$$

So $f$ is definable in the model $V[H]\left[g_{M}\right]$, and hence is in $V[H]\left[g_{M}\right]$.

The forcing poset $M \cap \mathbb{P}$ is countable. Since $\mathbb{Q}$ is $\omega_{1}$-closed and $\mathbb{P} \in V$, it follows that $M \cap \mathbb{P} \in V$. As $M \cap \mathbb{P}$ and $\mathbb{Q}$ are both in $V$, the product lemma implies that $V[H]\left[g_{M}\right]=V\left[g_{M}\right][H]$. As $M \cap \mathbb{P}$ is countable, it is $\omega_{1}$-c.c. So by Easton's lemma, $\mathbb{Q}$ is $\omega_{1}$-distributive in $V\left[g_{M}\right]$. But $f \in V[H]\left[g_{M}\right]=V\left[g_{M}\right][H]$ and $f$ is countable, so $f \in V\left[g_{M}\right]$. And $V\left[g_{M}\right]=V[G \cap M] \subseteq V[G]$, so $f \in V[G]$.

Corollary 2.4. The principle GMP together with $2^{\omega} \leq \omega_{2}$ is consistent with the existence of an $\omega_{1}$-distributive nowhere c.c.c. forcing poset of size $\omega_{1}$. In particular, GMP together with $2^{\omega} \leq \omega_{2}$ does not imply Todorčević's maximality principle.

Proof. Let $\kappa$ be a supercompact cardinal. Then there is a strongly proper forcing poset $\mathbb{P}$ which collapses $\kappa$ to become $\omega_{2}$, forces that $2^{\omega}=\omega_{2}$, and forces GMP. For example, let $\mathbb{P}$ be the forcing poset consisting of finite adequate sets of countable models, ordered by reverse inclusion. See Sections 6 and 7 of 1 for the details.

Let $G$ be a generic filter on $\mathbb{P}$. Let $\mathbb{Q}:=\operatorname{Add}\left(\omega_{1}\right)^{V}$. Then $\mathbb{Q}$ is a non-trivial forcing poset in $V[G]$. In $V,|\mathbb{Q}|=2^{\omega}<\kappa$, so in $V[G],|\mathbb{Q}|<\kappa=\omega_{2}$. Hence, $|\mathbb{Q}|=\omega_{1}$ in $V[G]$. In $V, \mathbb{P}$ is strongly proper on a stationary set and $\mathbb{Q}$ is $\omega_{1}$-closed. By Theorem 2.3, it follows that $\mathbb{Q}$ is $\omega_{1}$-distributive in $V[G]$.

We comment that in the model of [1, Section 7], GMP holds but there exists an $\omega_{1}$-Suslin tree. Since an $\omega_{1}$-Suslin tree is an example of a c.c.c., $\omega_{1}$-distributive forcing of size $\omega_{1}$, this provides a different proof that Todorčević's maximality principle does not follow from GMP.

Let us give another example of a strongly proper variation of a classical result.

Theorem 2.5. Let $2^{\omega}=\omega_{2}$ and suppose that $T$ is an $\omega_{2}$-Aronszajn tree. Assume that $\mathbb{Q}$ is an $\omega_{1}$-closed forcing poset which collapses $\omega_{2}$, and $\mathbb{P}$ is a forcing poset which is $\omega_{1}-$ Knaster in $V^{\mathbb{Q}}$. Then $\mathbb{P} \times \mathbb{Q}$ does not add a cofinal branch of $T$.

Proof. See [3, Lemma 23.1].

Theorem 2.6. Suppose that $T$ is a tree whose height is an ordinal with uncountable cofinality, all of whose levels have size less than $2^{\omega}$. Let $\mathbb{P}$ be strongly proper on a stationary set and $\mathbb{Q}$ be $\omega_{1}$-closed. Then $\mathbb{P} \times \mathbb{Q}$ does not add any new cofinal branches to $T$.

Proof. Let $G \times H$ be a $V$-generic filter on $\mathbb{P} \times \mathbb{Q}$. By the product lemma, $V[G \times H]=$ $V[H][G]$. By the proof of [3, Lemma 23.1], $\omega_{1}$-closed forcing cannot add new cofinal branches to $T$, so there are no new cofinal branches of $T$ in $V[H]$. By Theorem $2.2, \mathbb{P}$ is still strongly proper on a stationary set in $V[H]$, and hence has the $\omega_{1}$ approximation property in $V[H]$. Hence, $\mathbb{P}$ cannot add any new cofinal branches of $T$ over $V[H]$. Therefore, $V[H][G]=V[G \times H]$ has no new cofinal branches of $T$. 
Theorems 2.3 and 2.6 can be generalized to higher cardinals. First, the following generalization of Theorem 2.2 follows by a straightforward modification of the original argument given in [2, Theorem 5.5].

Theorem 2.7. Let $\kappa$ be a regular uncountable cardinal. Let $\mathbb{P}$ and $\mathbb{Q}$ be forcing posets, where $\mathbb{Q}$ is $\kappa$-closed. Suppose that for all large enough regular cardinals $\theta$, there are stationarily many internally approachable $N \in P_{\kappa}(H(\theta))$ with $N \cap \kappa \in \kappa$ such that $\mathbb{P}$ is strongly proper for $N$. Then $\mathbb{Q}$ forces that $\mathbb{P}$ is $\kappa$-strongly proper on a stationary set.

We need to work with internally approachable models $N$ in $P_{\kappa}(H(\theta))$ because those are the models for which $\kappa$-closed forcings have $N$-generic conditions.

Secondly, the proofs of Theorems 2.3 and 2.6 can be modified in the obvious way to prove the following results. We leave the details for the interested reader.

Theorem 2.8. Let $\kappa$ be a regular uncountable cardinal. Let $\mathbb{P}$ and $\mathbb{Q}$ be forcing posets, where $\mathbb{Q}$ is $\kappa$-closed. Suppose that for all large enough regular cardinals $\theta$, there are stationarily many internally approachable models $N \in P_{\kappa}(H(\theta))$ with $N \cap \kappa \in \kappa$ such that $\mathbb{P}$ is strongly proper for $N$. Then $\mathbb{P}$ forces that $\mathbb{Q}$ is $\kappa$ distributive.

Theorem 2.9. Let $\mu<\kappa$ be regular cardinals with $2^{<\mu}<\kappa$. Let $T$ be a tree whose height is an ordinal with cofinality at least $\kappa$ such that the levels of $T$ all have size less than $2^{\mu}$. Suppose that $\mathbb{P}$ and $\mathbb{Q}$ are forcing posets, where $\mathbb{Q}$ is $\kappa$-closed, and assume that for all large enough regular cardinals $\theta$, there are stationarily many internally approachable models $N \in P_{\kappa}(H(\theta))$ with $N \cap \kappa \in \kappa$ such that $\mathbb{P}$ is strongly proper for $N$. Then $\mathbb{P} \times \mathbb{Q}$ does not add new cofinal branches to $T$.

\section{WEAK APPROXIMATION AND GUESSING}

We now begin the main topic of the paper by introducing a weak form of guessing. We will show that the existence of stationarily many weakly guessing models implies many of the same strong consequences as the existence of stationarily many $\omega_{1}$ guessing models.

For the remainder of the paper, we will say that $N$ is an elementary substructure to mean that $N$ is an elementary substructure of some transitive set which models ZFC - Powerset and correctly computes $\omega_{1}$.

Definition 3.1. Let $N$ be set of size $\omega_{1}$ with $\omega_{1} \subseteq N$.

Let $\kappa \in N$ be an ordinal such that $N$ models that $\kappa$ is regular uncountable. We say that $N$ is weakly $\kappa$-guessing if whenever $f: \sup (N \cap \kappa) \rightarrow O n$ is a function such that for cofinally many $\alpha<\sup (N \cap \kappa), f \uparrow \alpha \in N$, then there is a function $g \in N$ with domain $\kappa$ such that $g\lceil\sup (N \cap \kappa)=f$.

We say that $N$ is weakly guessing if for any $\kappa \in N$ which $N$ models is regular uncountable, $N$ is weakly $\kappa$-guessing.

Note that in the case that $\kappa=\omega_{1}, N$ is weakly $\omega_{1}$-guessing iff whenever $f: \omega_{1} \rightarrow$ $O n$ is a function such that for all $\alpha<\omega_{1}, f\lceil\alpha \in N$, then $f \in N$.

Lemma 3.2. Let $N$ be an elementary substructure of size $\omega_{1}$ with $\omega_{1} \subseteq N$. Let $\kappa \in N$ be a regular uncountable cardinal, and assume that $N$ is weakly $\kappa$-guessing. Then $\operatorname{cf}(\sup (N \cap \kappa))=\omega_{1}$. 
Proof. Suppose for a contradiction that $b$ is a cofinal subset of $N \cap \kappa$ with order type $\omega$. Let $f: \sup (N \cap \kappa) \rightarrow 2$ be the function such that $f(\gamma)=1$ iff $\gamma \in b$. Then easily for all $\alpha \in N \cap \kappa, f\lceil\alpha \in N$, since $f\lceil\alpha$ is the characteristic function of the finite set $b \cap \alpha$.

Since $N$ is weakly $\kappa$-guessing, there is a function $g: \kappa \rightarrow O n$ in $N$ such that $g\lceil\sup (N \cap \kappa)=f$. For all $\alpha \in N \cap \kappa, g\lceil\alpha=f\lceil\alpha$ is the characteristic function of a finite set. So by the elementarity of $N$, for all $\alpha \in \kappa, g\lceil\alpha$ is the characteristic function of a finite set. In particular, $g\lceil\sup (N \cap \kappa)=f$ is the characteristic function of a finite set, which contradicts the fact that $b$ is infinite.

As with the property of being $\omega_{1}$-guessing, we can characterize the property of a set being weakly guessing in terms of an approximation property of its transitive collapse.

Definition 3.3. Let $W_{1}$ and $W_{2}$ be transitive sets or classes with $W_{1} \subseteq W_{2}$, and let $\lambda \in W_{1}$ be an ordinal. We say that the pair $\left(W_{1}, W_{2}\right)$ has the weak $\lambda$-approximation property if whenever $f: \lambda \rightarrow O n$ is a function in $W_{2}$ such that for all $\alpha<\lambda$, $f \mid \alpha \in W_{1}$, then $f \in W_{1}$.

Lemma 3.4. If $\left(W_{1}, W_{2}\right)$ has the $\omega_{1}$-approximation property, where $W_{1}$ is closed under intersections, then $\left(W_{1}, W_{2}\right)$ has the weak $\lambda$-approximation property for all ordinals $\lambda \in W_{1}$ with uncountable cofinality in $W_{1}$.

Proof. Let $f: \lambda \rightarrow O n$ be a function in $W_{2}$, where $W_{1} \models \operatorname{cf}(\lambda) \geq \omega_{1}$, and suppose that for all $\alpha<\lambda, f\left\lceil\alpha \in W_{1}\right.$. We claim that $f$ is in $W_{1}$. Since $\left(W_{1}, W_{2}\right)$ has the $\omega_{1}$-approximation property, it suffices to show that if $a$ is countable in $W_{1}$, then $a \cap f \in W_{1}$. Since $\lambda$ has uncountable cofinality in $W_{1}$, there is $\beta<\lambda$ such that $\operatorname{dom}(a) \cap \lambda \subseteq \beta$. Then $a \cap f=a \cap\left(f\lceil\beta)\right.$. Since $a$ and $f\left\lceil\beta\right.$ are in $W_{1}$, so is $a \cap f$.

Lemma 3.5. Let $N$ be an elementary substructure of size $\omega_{1}$ such that $\omega_{1} \subseteq N$. Let $\kappa \in N$ be a regular uncountable cardinal. Then $N$ is weakly $\kappa$-guessing iff the pair $\left(N_{0}, V\right)$ has the weak $\pi(\kappa)$-approximation property, where $\pi: N \rightarrow N_{0}$ is the transitive collapse of $N$.

Proof. Assume that $N$ is weakly $\kappa$-guessing, and we will prove that $\left(N_{0}, V\right)$ has the weak $\pi(\kappa)$-approximation property. By Lemma 3.2, $\operatorname{cf}(\sup (N \cap \kappa))=\omega_{1}$. Let $f: \pi(\kappa) \rightarrow O n$ be a function such that for all $\alpha<\pi(\kappa), f\left\lceil\alpha \in N_{0}\right.$. We will show that $f \in N_{0}$.

Define

$$
f^{*}:=\bigcup\left\{\pi^{-1}(f\lceil\alpha): \alpha<\pi(\kappa)\} .\right.
$$

It is easy to check that $f^{*}$ is an ordinal-valued function with domain $\sup (N \cap \kappa)$. For all $\beta \in N \cap \kappa, f^{*}\left\lceil\beta=\pi^{-1}(f\lceil\pi(\beta))\right.$, so for cofinally many $\alpha<\sup (N \cap \kappa)$, $f^{*}\lceil\alpha \in N$. Since $N$ is weakly $\kappa$-guessing, there is $g: \kappa \rightarrow O n$ in $N$ such that $g\left\lceil\sup (N \cap \kappa)=f^{*}\right.$. It is easy to check that $\pi(g)=f$, and hence $f \in N_{0}$.

Conversely, assume that $\left(N_{0}, V\right)$ has the weak $\pi(\kappa)$-approximation property, and we will prove that $N$ is weakly $\kappa$-guessing. Assume that $f: \sup (N \cap \kappa) \rightarrow O n$ is a function such that for cofinally many $\alpha<\sup (N \cap \kappa), f\lceil\alpha \in N$. Note that for all $\beta \in N \cap \kappa=N \cap \sup (N \cap \kappa), f \uparrow \beta \in N$.

Let

$$
f^{*}:=\bigcup\{\pi(f\lceil\alpha): \alpha \in N \cap \kappa\} .
$$


It is easy to check that $f^{*}$ is an ordinal-valued function with domain $\pi(\kappa)$, and for all $\beta<\pi(\kappa), f^{*}\left\lceil\beta=\pi\left(f\left\lceil\pi^{-1}(\beta)\right) \in N_{0}\right.\right.$. Since $\left(N_{0}, V\right)$ has the weak $\pi(\kappa)$ approximation property, $f^{*} \in N_{0}$. Then $\pi^{-1}\left(f^{*}\right) \in N$, and it is easy to check that $\pi^{-1}\left(f^{*}\right)\lceil\sup (N \cap \kappa)=f$.

Corollary 3.6. Let $N$ be an elementary substructure of size $\omega_{1}$ such that $\omega_{1} \subseteq$ $N$. If $N$ is $\omega_{1}$-guessing, then $N$ is weakly $\kappa$-guessing for all regular uncountable cardinals $\kappa \in N$.

Proof. Let $\pi: N \rightarrow N_{0}$ be the transitive collapse of $N$. Suppose that $N$ is $\omega_{1^{-}}$ guessing. Then by Lemma $1.5,\left(N_{0}, V\right)$ has the $\omega_{1}$-approximation property. Let $\kappa \in N$ be regular uncountable. Then $N_{0}$ models that $\pi(\kappa)$ is regular uncountable, and hence has uncountable cofinality. So $\left(N_{0}, V\right)$ has the weak $\pi(\kappa)$-approximation property by Lemma 3.4. By Lemma $3.5, N$ is weakly $\kappa$-guessing.

Definition 3.7. Let $\kappa$ be a regular uncountable cardinal. A forcing poset $\mathbb{P}$ is said to have the weak $\kappa$-approximation property if $\mathbb{P}$ forces that the pair $\left(V, V^{\mathbb{P}}\right)$ has the weak $\kappa$-approximation property.

Lemma 3.8. Let $\kappa$ be a regular uncountable cardinal. If $\mathbb{P}$ has the weak $\kappa$ approximation property, then $\mathbb{P}$ forces that $\operatorname{cf}(\kappa)>\omega$. In particular, if $\mathbb{P}$ has the weak $\omega_{1}$-approximation property, then $\mathbb{P}$ preserves $\omega_{1}$.

Proof. Similar to the proof of Lemma 3.2.

We can also define an indestructible version of a weakly guessing model.

Definition 3.9. Let $N$ be a set of size $\omega_{1}$ with $\omega_{1} \subseteq N$.

Let $\kappa \in N$ be a regular uncountable cardinal. We say that $N$ is indestructibly weakly $\kappa$-guessing if $N$ is weakly $\kappa$-guessing in any generic extension by an $\omega_{1}$ preserving forcing poset.

We say that $N$ is indestructibly weakly guessing if for all regular uncountable cardinals $\kappa \in N, N$ is indestructibly weakly $\kappa$-guessing.

With the idea of a weakly guessing model at hand, we can now introduce principles which weaken GMP and IGMP.

Definition 3.10. For a cardinal $\lambda \geq \omega_{2}$, let $w G M P(\lambda)$ be the statement that there exist stationarily many sets $N \in P_{\omega_{2}}(H(\lambda))$ such that $\omega_{1} \subseteq N$ and $N$ is weakly guessing. Let $w G M P$ be the statement that $w G M P(\lambda)$ holds for all cardinals $\lambda \geq \omega_{2}$.

Definition 3.11. For a cardinal $\lambda \geq \omega_{2}$, let $w \operatorname{IGMP}(\lambda)$ be the statement that there exist stationarily many sets $N \in P_{\omega_{2}}(H(\lambda))$ such that $\omega_{1} \subseteq N$ and $N$ is indestructibly weakly guessing. Let wIGMP be the statement that wIGMP $(\lambda)$ holds for all cardinals $\lambda \geq \omega_{2}$.

Corollary 3.12. Let $\lambda \geq \omega_{2}$. Then $\operatorname{GMP}(\lambda)$ implies $w G M P(\lambda)$, and $\operatorname{IGMP}(\lambda)$ implies wIGMP $(\lambda)$. Thus, GMP implies wGMP, and IGMP implies wIGMP.

Proof. Immediate from Corollary 3.6. 
We now explore strong consequences of the existence of weakly guessing models. The main point is that almost all of the known consequences of the existence of $\omega_{1}$-guessing models follow from the existence of weakly guessing models 1

It is straightforward to check that the following three consequences of the existence of $\omega_{1}$-guessing models follow from the existence of weakly $\omega_{1}$-guessing models, by slight modifications of the proofs given in [2].

Proposition 3.13. Suppose that there are stationarily many $N$ in $P_{\omega_{2}}\left(H\left(\omega_{3}\right)\right)$ such that $N$ is weakly $\omega_{1}$-guessing and $N \cap \omega_{2}$ has cofinality $\omega_{1}$. Then $\neg A P_{\omega_{1}}$.

Proof. The argument of [2, Proposition 2.6] shows that there exists a function $f: \omega_{1} \rightarrow N$ which is cofinal in $N \cap \omega_{2}$ all of whose initial segments are in $N$. Since $N$ is weakly $\omega_{1}$-guessing, $f \in N$. By elementarity, $\sup (\operatorname{ran}(f))=N \cap \omega_{2} \in N$, which is impossible.

Proposition 3.14. Suppose that there are stationarily many $N$ in $P_{\omega_{2}}\left(H\left(\omega_{2}\right)\right)$ which are weakly $\omega_{1}$-guessing. Then there does not exist a weak $\omega_{1}$-Kurepa tree. In particular, $\mathrm{CH}$ fails.

Proof. See [2, Proposition 2.8].

Proposition 3.15. Assume that $2^{\omega} \leq \omega_{2}$ and there are cofinally many sets $N$ in $P_{\omega_{2}}\left(H\left(\omega_{2}\right)\right)$ which are indestructibly weakly $\omega_{1}$-guessing. Then Todorčević's maximality principle holds.

Proof. See [2, Theorem 3.9].

Weiss [15, Section 2] proved that the principle ITP $\left(\omega_{2}\right)$ implies the non-existence of $\omega_{2}$-Aronszajn trees.

Proposition 3.16. Suppose that there exist stationarily many weakly $\omega_{2}-$ guessing models in $P_{\omega_{2}}\left(H\left(\omega_{3}\right)\right)$. Then there does not exist an $\omega_{2}$-Aronszajn tree.

Proof. Let $T$ be a tree of height $\omega_{2}$, all of whose levels have cardinality less than $\omega_{2}$. We will prove that there is a branch of $T$ with order type $\omega_{2}$. Without loss of generality, assume that $T$ has underlying set $\omega_{2}$. Then $T \in H\left(\omega_{3}\right)$. Fix $N$ in $P_{\omega_{2}}\left(H\left(\omega_{3}\right)\right)$ such that $N \prec H\left(\omega_{3}\right), T \in N$, and $N$ is weakly $\omega_{2}$-guessing. Let $\alpha:=N \cap \omega_{2}$. By the elementarity of $N$, any node in $T$ of height less than $\alpha$ is in $N$.

Fix a node $y$ on level $\alpha$ of $T$. Define a function $f: \alpha \rightarrow \omega_{2}$ by letting $f(\beta)$ be the member of the set $\left\{x \in T: x<_{T} y\right\}$ which is on level $\beta$ of $T$. Then by elementarity, for all $\gamma<\alpha, f \uparrow \gamma$ is in $N$, since this function just enumerates in $<_{T}$-increasing order the elements of $T$ which are $<_{T}$-below $f(\gamma)$. Since $N$ is weakly $\omega_{2}$-guessing, there is a function $g: \omega_{2} \rightarrow O n$ in $N$ such that $g\lceil\alpha=f$.

We claim that the range of $g$ is a cofinal branch of $T$ of order type $\omega_{2}$, which completes the proof. For all $\gamma<\beta$ in $N \cap \omega_{2}=\alpha, g(\gamma)<_{T} g(\beta)$, where $g(\gamma)$ is on level $\gamma$ of $T$ and $g(\beta)$ is on level $\beta$ of $T$. By elementarity, the same statement holds for all $\gamma<\beta$ in $\omega_{2}$. It follows that the range of $g$ is a chain of $T$ which meets each level of $T$, and thus is a cofinal branch of $T$ of order type $\omega_{2}$.

\footnotetext{
${ }^{1} \mathrm{~A}$ possible exception is the result of Viale [13, Section 7.2] that the existence of stationarily many $\omega_{1}$-guessing models which are internally unbounded implies $\mathrm{SCH}$. We do not know whether $\mathrm{SCH}$ follows from stationarily many internally unbounded weakly guessing models.
} 
The square principle described in the next proposition was originally shown to fail under $\operatorname{ITP}\left(\omega_{2}\right)$ by Weiss [15, Section 4].

Proposition 3.17. Let $\kappa \geq \omega_{2}$ be regular, $\theta>\kappa$, and assume that there are stationarily many $N \in P_{\omega_{2}}(H(\theta))$ with bounded uniform cofinality $\omega_{1}$ which are weakly $\kappa$-guessing. Then there does not exist a sequence

$$
\left\langle c_{\alpha}: \alpha \in \kappa \cap \operatorname{cof}\left(\omega_{1}\right)\right\rangle
$$

satisfying:

(1) $c_{\alpha}$ is a club subset of $\alpha$;

(2) for any ordinal $\gamma<\kappa$, the set

$$
E_{\gamma}:=\left\{c_{\alpha} \cap \gamma: \alpha \in \kappa \cap \operatorname{cof}\left(\omega_{1}\right), \gamma \in \lim \left(c_{\alpha}\right)\right\}
$$

has size less than $\omega_{2}$;

(3) there is no club set $D \subseteq \kappa$ such that for every $\gamma \in \lim (D) \cap \kappa, D \cap \gamma \in E_{\gamma}$.

Proof. Suppose for a contradiction that

$$
\vec{c}=\left\langle c_{\alpha}: \alpha \in \kappa \cap \operatorname{cof}\left(\omega_{1}\right)\right\rangle
$$

is such a sequence. Fix $N \in P_{\omega_{2}}(H(\theta))$ such that $N \prec(H(\theta), \in, \kappa, \vec{c})$ and $N$ is weakly $\kappa$-guessing. Note that for all $\gamma \in N \cap \kappa, E_{\gamma} \in N$, and so $E_{\gamma} \subseteq N$ since $\left|E_{\gamma}\right|<\omega_{2}$ and $\omega_{1} \subseteq N$.

Let $\alpha:=\sup (N \cap \kappa)$. Since $\kappa$ is regular, $\alpha<\kappa$. By Lemma 3.2, $\operatorname{cf}(\alpha)=\omega_{1}$.

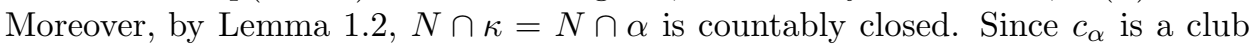
subset of $\alpha$, it follows that $\lim \left(c_{\alpha}\right) \cap N \cap \alpha$ is cofinal in $\alpha$.

Let $f: \alpha \rightarrow 2$ be the characteristic function of $c_{\alpha}$. We claim that there are cofinally many $\gamma<\alpha$ such that $f\left\lceil\gamma\right.$ is in $N$. The set $\lim \left(c_{\alpha}\right) \cap N \cap \alpha$ is cofinal in $\alpha$. Consider $\gamma$ in this set. Then $c_{\alpha} \cap \gamma \in E_{\gamma}$. Since $E_{\gamma} \subseteq N$ as observed above, $c_{\alpha} \cap \gamma \in N$. But $f\left\lceil\gamma\right.$ is just the characteristic function of $c_{\alpha} \cap \gamma$, and hence is in $N$.

Since $N$ is weakly $\kappa$-guessing, there is a function $g: \kappa \rightarrow O n$ in $N$ such that $g\left\lceil\alpha=f\right.$. Let $D:=\{\gamma<\kappa: g(\gamma)=1\}$. Then $D \cap \alpha=c_{\alpha}$. An easy argument using elementarity and the fact that $c_{\alpha}$ is a club in $\alpha$ shows that $D$ is a club subset of $\kappa$.

If $\gamma$ is a limit point of $D$ in $N \cap \kappa=N \cap \alpha$, then $\gamma$ is a limit point of $c_{\alpha}$, and hence $c_{\alpha} \cap \gamma=D \cap \gamma$ is in $E_{\gamma}$. By elementarity, it follows that if $\gamma$ is a limit point of $D$ in $\kappa$, then $D \cap \gamma \in E_{\gamma}$. This contradicts property 3 of the sequence $\vec{c}$.

Corollary 3.18. The principle wGMP implies $\neg \square$ $\square_{\kappa}$ and $\neg \square(\lambda)$ for all cardinals $\kappa \geq \omega_{1}$ and regular cardinals $\lambda \geq \omega_{2}$.

\section{Guessing MOdels And COVERING}

In this section we will prove that $\mathrm{GMP}\left(\omega_{2}\right)$ implies the existence of stationarily many $\omega_{1}$-guessing models in $P_{\omega_{2}}\left(H\left(\omega_{2}\right)\right)$ which are not internally unbounded.

Lemma 4.1. Suppose that $\left\langle M_{n}: n<\omega\right\rangle$ is a $\subseteq$-increasing sequence of sets which are weakly $\omega_{1}$-guessing. Then $M:=\bigcup\left\{M_{n}: n<\omega\right\}$ is weakly $\omega_{1}$-guessing.

Proof. Let $f: \omega_{1} \rightarrow O n$ be a function such that for cofinally many $\alpha<\omega_{1}$, $f\left\lceil\alpha \in M\right.$. Note that since $\omega_{1} \subseteq M$, this implies that for all $\alpha<\omega_{1}, f\lceil\alpha \in M$. We will prove that $f \in M$. 
As $M=\bigcup\left\{M_{n}: n<\omega\right\}$, for each $\alpha<\omega_{1}$ we can fix $n_{\alpha}<\omega$ such that $f \uparrow \alpha \in M_{n_{\alpha}}$. Fix an uncountable set $X \subseteq \omega_{1}$ and $n<\omega$ such that for all $\alpha \in X$, $n_{\alpha}=n$.

Since $X$ is uncountable, it is unbounded in $\omega_{1}$. Therefore, $\bigcup\{f \uparrow \alpha: \alpha \in X\}=f$. For each $\alpha \in X, n_{\alpha}=n$ implies that $f \uparrow \alpha \in M_{n}$. So there are cofinally many $\alpha<\omega_{1}$ such that $f \uparrow \alpha \in M_{n}$. Since $M_{n}$ is weakly $\omega_{1}$-guessing, $f \in M_{n}$. As $M_{n} \subseteq M, f \in M$, and we are done.

Corollary 4.2. Let $\theta \geq \omega_{2}$ be a regular cardinal. Assume that there are stationarily many $M \in P_{\omega_{2}}(H(\theta))$ such that $M$ is weakly $\omega_{1}$-guessing. Then there are stationarily many $M \in P_{\omega_{2}}(H(\theta))$ such that $M$ is weakly $\omega_{1}$-guessing and for all regular cardinals $\lambda \geq \omega_{2}$ in $M \cup\{\theta\}, \sup (M \cap \lambda)=\omega$.

Proof. Let $F: H(\theta)^{<\omega} \rightarrow H(\theta)$ be a function. Since there are stationarily many $N \in P_{\omega_{2}}(H(\theta))$ which are weakly $\omega_{1}$-guessing, we can inductively define a sequence $\left\langle M_{n}: n<\omega\right\rangle$ such that for all $n<\omega, M_{n} \prec H(\theta), M_{n}$ is closed under $F, M_{n}$ is weakly $\omega_{1}$-guessing, and $M_{n} \in M_{n+1}$.

Let $M:=\bigcup\left\{M_{n}: n<\omega\right\}$. By Lemma $4.1, M$ is weakly $\omega_{1}$-guessing. For any regular cardinal $\lambda$ in $M \cup\{\theta\}$, the countable set $\left\{\sup \left(M_{n} \cap \lambda\right): n<\omega\right\}$ is cofinal in $\sup (M \cap \lambda)$, and hence $\operatorname{cf}(\sup (M \cap \lambda))=\omega$.

In particular, being a weakly $\omega_{1}$-guessing model does not imply being internally unbounded.

Lemma 4.3. Let $M \in P_{\omega_{2}}\left(H\left(\omega_{2}\right)\right)$ be a set such that $\omega_{1} \subseteq M$ and $M \prec H\left(\omega_{2}\right)$. Then $M$ is $\omega_{1}$-guessing iff $M$ is weakly $\omega_{1}$-guessing.

Proof. By Corollary 3.6, if $M$ is $\omega_{1}$-guessing, then $M$ is weakly $\omega_{1}$-guessing. Assume that $M$ is weakly $\omega_{1}$-guessing, and we will show that $M$ is $\omega_{1}$-guessing.

Let $X$ be a bounded subset of $M \cap O n=M \cap \omega_{2}$ such that for any countable set $a \in M, a \cap X \in M$. We will show that $X \in M$. If $X$ is a bounded subset of $\omega_{1}$, then we can fix a countable ordinal $\beta$ with $\sup (X)<\beta$. Then by assumption, $X=X \cap \beta$ is in $M$. So assume that $\sup (X) \geq \omega_{1}$.

Since $\sup (X)<\sup \left(M \cap \omega_{2}\right)$, fix $\alpha \in M \cap \omega_{2}$ such that $\sup (X)<\alpha$. By elementarity, fix a bijection $f: \omega_{1} \rightarrow \alpha$ in $M$. Then $Y:=f^{-1}(X)$ is a subset of $\omega_{1}$. Let $g: \omega_{1} \rightarrow 2$ be the characteristic function of $Y$. We claim that for all $\beta<\omega_{1}$, $g \uparrow \beta \in M$. If $\beta<\omega_{1}$, then $f[\beta]$ is a countable set in $M$, and therefore $f[\beta] \cap X$ is in $M$. Hence, $f^{-1}(f[\beta] \cap X)=Y \cap \beta$ is in $M$. Since $g \uparrow \beta$ is the characteristic function of $Y \cap \beta, g\lceil\beta \in M$.

As $M$ is weakly $\omega_{1}$-guessing, it follows that $g$ is in $M$. As $g$ is the characteristic function of $Y, Y \in M$. By elementarity, $f[Y]=X$ is in $M$.

Corollary 4.4. The principles $\operatorname{GMP}\left(\omega_{2}\right)$ and $w G M P\left(\omega_{2}\right)$ are equivalent.

Proof. Immediate from Lemma 4.3.

Corollary 4.5. Assuming $G M P\left(\omega_{2}\right)$, there are stationarily many $M \in P_{\omega_{2}}\left(H\left(\omega_{2}\right)\right)$ such that $\sup \left(M \cap \omega_{2}\right)$ has cofinality $\omega$ and $M$ is $\omega_{1}$-guessing (and in particular, $M$ is not internally unbounded).

Proof. Immediate from Corollary 4.2 and Lemma 4.3. 


\section{FORCING AXIOMS AND WEAK GUESSING}

The goal of the rest of the paper is to prove the consistency of the existence of stationarily many indestructibly weakly guessing models $N$ which have a countable bounded subset which is not covered by any countable set in $N$. We will use the following theorem of Woodin.

Theorem 5.1 (Woodin [16]). Let $\mathbb{P}$ be a forcing poset, and assume that for any family of $\omega_{1}$ many dense subsets of $\mathbb{P}$, there exists a filter on $\mathbb{P}$ which meets each set in the family. Then for any regular cardinal $\theta$ with $\mathbb{P} \in H(\theta)$, there are stationarily many $N \in P_{\omega_{2}}(H(\theta))$ with $\omega_{1} \subseteq N$ for which there exists an $N$-generic filter on $\mathbb{P}$.

Recall that a filter $G$ on $\mathbb{P}$ is $N$-generic if for any dense set $D \in N, D \cap G \cap N \neq \emptyset$.

Lemma 5.2. Let $\mathbb{P}$ be a forcing poset, $\theta \geq \omega_{2}$ a regular cardinal with $\mathbb{P} \in H(\theta)$, and $N \in P_{\omega_{2}}(H(\theta))$ such that $\omega_{1} \subseteq N$ and $N \prec(H(\theta), \in, \mathbb{P})$. Suppose that $G$ is an $N$-generic filter on $\mathbb{P}$. Assume that $p \in G, \lambda \in N$, and $p$ forces that there is a countable subset of $\lambda$ which is not covered by any countable set in $V$. Then there is a countable subset of $N \cap \lambda$ which is not covered by any countable set in $N$.

Proof. Suppose that $p$ forces that $\dot{a}$ is a countable subset of $\lambda$ which is not covered by any countable set in $V$. By elementarity and the fact that $G$ is an $N$-generic filter, without loss of generality we may assume that $p$ and $\dot{a}$ are in $N$. By elementarity, we can fix a $\mathbb{P}$-name $\dot{f}$ in $N$ which $p$ forces is a surjection of $\omega$ onto $\dot{a}$. Now let $b$ be the set of $\beta \in N$ such that for some $q \in G \cap N$ and $n<\omega, q \Vdash \dot{f}(n)=\check{\beta}$. Since $G$ is a filter, each $n$ has a unique such ordinal $\beta$, and hence $b$ is a countable subset of $N \cap \lambda$.

Let $c$ be a countable set in $N$, and we will show that $b$ is not a subset of $c$. Note that $p$ forces that $\dot{a}$ is not a subset of $c$. Let $D$ be the dense set of conditions which are either incompatible with $p$, or below $p$ and decide for some $n$ and $\beta$ that $\dot{f}(n)=\beta \in \dot{a} \backslash c$. By elementarity, $D \in N$. Since $G$ is $N$-generic, fix $q \in D \cap G \cap N$. Then $q \leq p$, and for some $n$ and $\beta$ in $N, q \Vdash \dot{f}(n)=\beta \in \dot{a} \backslash c$. Then $\beta \in b \backslash c$, and we are done.

Lemma 5.3. Let $\mathbb{P}$ be a forcing poset, $\theta \geq \omega_{2}$ a regular cardinal with $\mathbb{P} \in H(\theta)$, and $N \in P_{\omega_{2}}(H(\theta))$ such that $\omega_{1} \subseteq N$ and $N \prec(H(\theta), \in, \mathbb{P})$. Suppose that $G$ is an $N$-generic filter on $\mathbb{P}$. Assume that $p \in G, \lambda \in N$, and $p$ forces that there is a countable subset a of $\lambda$ such that for any countable set $c$ in $V, a \cap c$ is finite. Then there is a countable subset $b$ of $N \cap \lambda$ such that for any countable set $c$ in $N, b \cap c$ is finite.

Proof. Similar to the proof of Lemma 5.2.

In previous work, Krueger [7] used Woodin's theorem to prove that PFA implies the existence of stationarily many models which are internally club but not internally approachable. The proof involved specializing a certain tree of height and size $\omega_{1}$ which was built on a model $N$. Later, Viale-Weiss [14] expanded on Krueger's application of Woodin's theorem to produce $\omega_{1}$-guessing models.

The next result was originally proven in [14 for forcing posets which have the $\omega_{1}$ approximation property and models which are $\omega_{1}$-guessing. We have modified the argument to handle forcing posets which have the weak $\kappa$-approximation property and models which are indestructibly weakly $\kappa$-guessing. 
Proposition 5.4. Let $\lambda \geq \omega_{2}$ be a regular cardinal and $A \subseteq \lambda$ a set of regular uncountable cardinals. Assume that $\mathbb{P}$ is a forcing poset which has the weak $\kappa$ approximation property for all $\kappa \in A$ and forces that $\left(2^{\lambda}\right)^{V}$ has size $\omega_{1}$. Then there exist a set $w$ and a $\mathbb{P}$-name $\dot{\mathbb{Q}}$ for an $\omega_{1}$-c.c. forcing poset satisfying: for any regular cardinal $\chi$ with $\mathbb{P}, \lambda, w$, and $\dot{\mathbb{Q}}$ in $H(\chi)$, for any set $M \in P_{\omega_{2}}(H(\chi))$ such that $\omega_{1} \subseteq M$ and $M \prec(H(\chi), \in, \mathbb{P} * \dot{\mathbb{Q}}, \lambda, A, w)$, if there exists an $M$-generic filter on $\mathbb{P} * \dot{\mathbb{Q}}$, then $M \cap H(\lambda)$ is indestructibly weakly $\kappa$-guessing for all $\kappa \in A \cap M$.

Proof. We begin by fixing a generic filter $G$ on $\mathbb{P}$, and analyze what happens in $V[G]$. We will define a tree $\left(T,<_{T}\right)$ in $V[G]$ of height and size $\omega_{1}$ which has at most $\omega_{1}$ many uncountable branches, together with subtrees $T^{0}$ and $T^{1}$, where $T^{1}$ has no uncountable branches. We then let $\mathbb{Q}$ be the standard $\omega_{1}$-c.c. forcing poset for adding a specializing function to the tree $T^{1}$. The tree $T$ will be the disjoint sum of trees $\left(T_{\kappa},<_{\kappa}\right)$, for $\kappa \in A$.

Working in $V[G]$, consider $\kappa \in A$. Since $\mathbb{P}$ has the $\kappa$-approximation property, it forces that $\operatorname{cf}(\kappa)>\omega$ by Lemma 3.8. As $\mathbb{P}$ collapses $\lambda$ to have size $\omega_{1}, \mathbb{P}$ forces that $\operatorname{cf}(\kappa)=\omega_{1}$. Fix a sequence $\left\langle\beta_{i}^{\kappa}: i<\omega_{1}\right\rangle$ which is increasing and cofinal in $\kappa$. Let $T_{\kappa}$ denote the set of functions in $V$ whose domain is equal to $\beta_{i}^{\kappa}$ for some $i<\omega_{1}$, and whose range is a subset of $\lambda$. For $f$ and $g$ in $T_{\kappa}$, let $f<_{\kappa} g$ if $f$ is a proper subset of $g$. Clearly $\left(T_{\kappa},<_{\kappa}\right)$ is a tree. Since $\left(2^{\lambda}\right)^{V}$ has size $\omega_{1}$ in $V[G]$, the tree $\left(T_{\kappa},<_{\kappa}\right)$ has height and size $\omega_{1}$.

Let $B_{\kappa}$ be the set of all functions $f: \kappa \rightarrow \lambda$ such that for all $i<\omega_{1}, f\left\lceil\beta_{i}^{\kappa} \in T_{\kappa}\right.$. Note that if $f \in B_{\kappa}$, then since $T_{\kappa} \subseteq V$, it follows that for all $\alpha<\kappa, f\lceil\alpha \in V$. Since $\mathbb{P}$ has the weak $\kappa$-approximation property, it follows that $B_{\kappa} \subseteq V$. So in fact $B_{\kappa}$ is the set of all functions in $V$ from $\kappa$ into $\lambda$. Since $\left(2^{\lambda}\right)^{V}$ has size $\omega_{1}, B_{\kappa}$ has size $\omega_{1}$. Note that if $X$ is an uncountable branch of $T_{\kappa}$, then $\bigcup X \in B_{\kappa}$. It follows that $T_{\kappa}$ has at most $\omega_{1}$ many uncountable branches.

Let $\left(T,<_{T}\right)$ be the disjoint sum of such trees. So elements of $T$ are pairs of the form $(\kappa, g)$, where $\kappa \in A$ and $g \in T_{\kappa}$, and $\left(\kappa_{0}, g\right)<_{T}\left(\kappa_{1}, h\right)$ if $\kappa_{0}=\kappa_{1}$ and $g<_{\kappa_{0}} h$. Since $\lambda$ has size $\omega_{1}$ in $V[G], T$ is a tree of height and size $\omega_{1}$. Since any uncountable branch of $T$ obviously yields an uncountable branch in some tree $T_{\kappa}, T$ has at most $\omega_{1}$ many uncountable branches.

Let $B=\bigcup\left\{B_{\kappa}: \kappa \in A\right\}$. Then $B$ has size at most $\omega_{1}$. So it is straightforward to define an injective function $g: B \rightarrow T$ such that for all $b \in B, g(b)=\left(\kappa, b\left\lceil\beta_{i}^{\kappa}\right)\right.$ for some $i<\omega_{1}$, where $b \in B_{\kappa}$. For example, enumerate $B$ as $\left\langle d_{i}: i<\omega_{1}\right\rangle$ and let $g\left(d_{i}\right):=\left(\kappa, d_{i}\left\lceil\beta_{i}^{\kappa}\right)\right.$, where $d_{i} \in B_{\kappa}$.

Now define subtrees $T^{0}$ and $T^{1}$ of $T$ by

$$
T^{0}:=\left\{(\kappa, t) \in T: \exists b \in B_{\kappa}\left(g(b)<_{T}(\kappa, t), t \subseteq b\right)\right\}
$$

and

$$
T^{1}:=T \backslash T^{0} .
$$

Then $T^{1}$ is a tree of height and size less than or equal to $\omega_{1}$.

We claim that $T^{1}$ has no uncountable branch. Suppose for a contradiction that $X$ is an uncountable branch of $T^{1}$. Then for some $\kappa \in A$,

$$
b:=\bigcup\{f:(\kappa, f) \in X\}
$$

is in $B_{\kappa}$. Fix $i<\omega_{1}$ such that $g(b)=\left(\kappa, b\left\lceil\beta_{i}^{\kappa}\right)\right.$. Since $X$ is uncountable, there is $j>i$ such that $\left(\kappa, b \uparrow \beta_{j}^{\kappa}\right)$ is in $X$. Then

$$
g(b)=\left(\kappa, b \uparrow \beta_{i}^{\kappa}\right)<_{T}\left(\kappa, b \uparrow \beta_{j}^{\kappa}\right)
$$


and $b \uparrow \beta_{j}^{\kappa} \subseteq b$. But then by the definition of $T^{0}, b \uparrow \beta_{j}^{\kappa} \in T^{0}$. This contradicts that $X \subseteq T^{1}$.

In summary, $T^{1}$ is a tree with height and size less than or equal to $\omega_{1}$ which has no uncountable branches. Let $\mathbb{Q}$ be the standard $\omega_{1}$-c.c. forcing poset which adds a specializing function to $T^{1}$.

Now let us go back and consider $\kappa \in A$ in $V[G]$. Define $g_{\kappa}: B_{\kappa} \rightarrow T_{\kappa}$ by letting $g_{\kappa}(b)$ be the unique $t$ such that $g(b)=(\kappa, t)$. Then $g_{\kappa}$ is injective, and $g_{\kappa}(b)$ is equal to $b\left\lceil\beta_{i}^{\kappa}\right.$ for some $i<\omega_{1}$.

Define subtrees $T_{\kappa}^{0}$ and $T_{\kappa}^{1}$ of $T_{\kappa}$ by

$$
T_{\kappa}^{0}:=\left\{t:(\kappa, t) \in T^{0}\right\}
$$

and

It is easy to check that

$$
T_{\kappa}^{1}:=\left\{t:(\kappa, t) \in T^{1}\right\} .
$$

$$
T_{\kappa}^{0}=\left\{t \in T_{\kappa}: \exists b \in B_{\kappa}\left(g_{\kappa}(b)<_{\kappa} t \subseteq b\right)\right\},
$$

and

$$
T_{\kappa}^{1}=T_{\kappa} \backslash T_{\kappa}^{0} .
$$

Note that if $\dot{h}$ is a $\mathbb{Q}$-name for the specializing function on $T^{1}$ which was added by $\mathbb{Q}$, then $\mathbb{Q}$ forces that the function $\dot{h}_{\kappa}: T_{\kappa}^{1} \rightarrow \omega$ defined by $\dot{h}_{\kappa}(t)=\dot{h}(\kappa, t)$ is a specializing function on $T_{\kappa}^{1}$.

This completes our analysis of the forcing extension $V[G]$. Since $G$ was arbitrary, we can fix $\mathbb{P}$-names $\dot{T}, \dot{<}_{T}, \dot{B}, \dot{g}, \dot{T}^{0}, \dot{T}^{1}$, and $\dot{\mathbb{Q}}$ such that $\mathbb{P}$ forces that these names satisfy the definitions which we made of these objects in $V^{\mathbb{P}}$ above. Moreover, we can fix a function which associates to each $\kappa \in A$ a set of $\mathbb{P}$-names $\left\langle\dot{\beta}_{i}^{\kappa}: i<\omega_{1}\right\rangle, \dot{T}_{\kappa}, \dot{<}_{\kappa}$, $\dot{B}_{\kappa}, \dot{g}_{\kappa}, \dot{T}_{\kappa}^{0}$, and $\dot{T}_{\kappa}^{1}$ such that $\mathbb{P}$ forces that these objects satisfy the definitions which we made of them in $V^{\mathbb{P}}$ above. Finally, let $\dot{h}$ be a $\mathbb{P} * \dot{\mathbb{Q}}$-name for the specializing function on $\dot{T}^{1}$ which is added by $\dot{\mathbb{Q}}$, and fix a function which associates to each $\kappa \in A$ a $\mathbb{P} * \dot{\mathbb{Q}}$-name for the function $\dot{h}_{\kappa}$ which specializes $\dot{T}_{\kappa}^{1}$. Now let $w$ be the set consisting of these finitely many names and functions.

Let $\chi$ be a regular cardinal such that $\mathbb{P}, \lambda, w$, and $\dot{\mathbb{Q}}$ are in $H(\chi)$. Let $M \in$ $P_{\omega_{2}}(H(\chi))$ be a set such that $\omega_{1} \subseteq M, M \prec(H(\chi), \in, \mathbb{P} * \dot{\mathbb{Q}}, \lambda, A, w)$, and there exists an $M$-generic filter $J$ on $\mathbb{P} * \dot{\mathbb{Q}}$. We will prove that $M \cap H(\lambda)$ is indestructibly weakly $\kappa$-guessing for all $\kappa \in A \cap M$. Let $G:=\{p: \exists \dot{q}(p, \dot{q}) \in J\}$. It is easy to check that $G$ is an $M$-generic filter on $\mathbb{P}$.

Consider $\kappa \in A \cap M$. Let $\theta:=\sup (M \cap \kappa)$. Suppose that $c: \theta \rightarrow \lambda$ is a function in an $\omega_{1}$-preserving generic extension $W$ such that for cofinally many $\alpha<\theta, c \mid \alpha \in M \cap H(\lambda)$. We will prove that for some function $c^{*}: \kappa \rightarrow \lambda$ in $M \cap H(\lambda), c^{*}\lceil\theta=c$. Note that since $\lambda$ is regular, any function mapping from $\kappa$ into $\lambda$ in $M$ is in $H(\lambda)$. So it suffices to find such a function $c^{*}$ in $M$.

For each $i<\omega_{1}$, let $\beta_{i}^{\kappa}$ be the unique ordinal such that for some $q \in G, q$ forces that $\dot{\beta}_{i}^{\kappa}=\check{\beta}_{i}^{\kappa}$. Since $G$ is a filter which is $M$-generic, it is straightforward to check that the sequence $\left\langle\beta_{i}^{\kappa}: i<\omega_{1}\right\rangle$ is increasing and cofinal in $M \cap \kappa$. For simplicity in notation, let us write $\beta_{i}:=\beta_{i}^{\kappa}$ for all $i<\omega_{1}$.

Given any $\mathbb{P}$-name $\dot{a}$ in $M$, we can interpret $\dot{a}$ by $G$ as the set of $x \in M$ such that for some $p \in G \cap M, p \Vdash \check{x} \in \dot{a}$. This gives us objects $T_{\kappa},<_{\kappa}, B_{\kappa}, g_{\kappa}, T_{\kappa}^{0}$, and 
$T_{\kappa}^{1}$ which interpret the $\mathbb{P}$-names $\dot{T}_{\kappa}, \dot{<}_{\kappa}, \dot{B}_{\kappa}, \dot{g}_{\kappa}, \dot{T}_{\kappa}^{0}$, and $\dot{T}_{\kappa}^{1}$. Similarly, interpret the $\mathbb{P} * \dot{\mathbb{Q}}$-name $\dot{h}_{\kappa}$ as $h_{\kappa}$. Using the $M$-genericity of $G$ and $J$ and arguments similar to those of Lemma 5.2, the following facts can be easily checked:

(1) $\left(T_{\kappa},<_{\kappa}\right)$ is the tree of all functions in $M$ whose domain is equal to $\beta_{i}$ for some $i<\omega_{1}$, and mapping into $\lambda$, ordered by $f<_{\kappa} g$ if $f$ is a proper subset of $g$;

(2) $B_{\kappa}$ is the set of all functions in $M$ of the form $b: \kappa \rightarrow \lambda$;

(3) $g_{\kappa}: B_{\kappa} \rightarrow T_{\kappa}$ is injective and $g_{\kappa}(b) \subseteq b$ for all $b \in B_{\kappa}$;

(4) $T_{\kappa}^{0}$ is the set of all $t \in T_{\kappa}$ such that for some $b \in B_{\kappa}, g_{\kappa}(b)<_{\kappa} t \subseteq b$;

(5) $T_{\kappa}^{1}=T_{\kappa} \backslash T_{\kappa}^{0}$

(6) $h_{\kappa}: T_{\kappa}^{1} \rightarrow \omega$ is a function such that whenever $f<_{\kappa} g$ are in $T_{\kappa}^{1}$, then $h_{\kappa}(f) \neq h_{\kappa}(g)$.

Recall that $c: \theta \rightarrow \lambda$ is a function in $W$ such that for cofinally many $\alpha<\theta$, $c \uparrow \alpha \in M$. In particular, for all $i<\omega_{1}, c \uparrow \beta_{i} \in T_{\kappa}$. Hence, the set $X:=\left\{c \uparrow \beta_{i}\right.$ : $\left.i<\omega_{1}\right\}$ is an uncountable branch of $T_{\kappa}$ in $W$. Since the function $h_{\kappa}$ is injective on chains, it is injective on $X \cap T_{\kappa}^{1}$. As $h_{\kappa}$ maps into $\omega$, there must exist $\gamma<\omega_{1}$ such that for all $\gamma \leq i<\omega_{1}, c \uparrow \beta_{i}$ is in $T_{\kappa}^{0}$.

Now $T_{\kappa}^{0}$ is the set of all $t \in T_{\kappa}$ such that for some $b \in B_{\kappa}, g_{\kappa}(b)<_{\kappa} t \subseteq b$. So for all $\gamma \leq i<\omega_{1}$, we can fix $b_{i} \in B_{\kappa}$ such that

$$
g_{\kappa}\left(b_{i}\right)<_{\kappa} c \uparrow \beta_{i} \subseteq b_{i} .
$$

In particular, $\operatorname{dom}\left(g_{\kappa}\left(b_{i}\right)\right)<\beta_{i}$, so fix $\zeta_{i}<i$ such that $\operatorname{dom}\left(g_{\kappa}\left(b_{i}\right)\right)=\beta_{\zeta_{i}}$. By Fodor's lemma applied in $W$, fix a stationary set $S \subseteq \omega_{1} \backslash \gamma$ in $W$ and $\zeta<\omega_{1}$ such that for all $i \in S, \zeta_{i}=\zeta$, and hence $\operatorname{dom}\left(g_{\kappa}\left(b_{i}\right)\right)=\beta_{\zeta}$.

It immediately follows that for all $i \in S$,

$$
g_{\kappa}\left(b_{i}\right)=b_{i} \uparrow \beta_{\zeta_{i}}=b_{i} \uparrow \beta_{\zeta}=\left(c \uparrow \beta_{i}\right) \uparrow \beta_{\zeta}=c \uparrow \beta_{\zeta} .
$$

Thus, for all $i<j$ in $S$,

$$
g_{\kappa}\left(b_{i}\right)=c \uparrow \beta_{\zeta}=g_{\kappa}\left(b_{j}\right)
$$

But $g_{\kappa}$ is injective. Hence, for all $i<j$ in $S, b_{i}=b_{j}$. Let $b:=b_{i}$ for some (any) $i \in S$. Then for all $i \in S, c \uparrow \beta_{i} \subseteq b$. Since $S$ is cofinal in $\omega_{1}$, it follows that $c \subseteq b$. So $b\lceil\theta=c$. Since $b \in M$, we are done.

\section{NAMBA FORCING}

In order to apply the results from the previous section, we will need to find a forcing poset which satisfies instances of the weak approximation property and yet adds a countable set of ordinals which is not covered by any countable set in the ground model. In this section we will define a Namba forcing which satisfies these requirements.

Let us introduce some notation which we will use in this section. By a tree of finite sequences we mean any set of finite sequences which is closed under initial segments. For finite sequences $\eta$ and $\nu$, we write $\eta \unlhd \nu$ to express that $\eta$ is an initial segment of $\nu$, and $\eta \triangleleft \nu$ to express that $\eta$ is a proper initial segment of $\nu$. We say that $\eta$ and $\nu$ are comparable if either $\eta \unlhd \nu$ or $\nu \unlhd \eta$; otherwise, they are incomparable.

Let $T$ be a tree of finite sequences. The elements of $T$ are called nodes of $T$. For $\eta \in T$, we write $\operatorname{Suc}_{T}(\eta)$ for the set $\left\{x: \eta^{\widehat{ }} x \in T\right\}$. A node $\eta$ of $T$ is a splitting 
node if $\left|\operatorname{Suc}_{T}(\eta)\right|>1$. We let $T_{\eta}$ denote the set of nodes in $T$ which are comparable with $\eta$. Note that $T_{\eta}$ is a tree of finite sequences and is a subset of $T$.

For the remainder of the section, fix a sequence $\left\langle\kappa_{n}: n<\omega\right\rangle$ of regular cardinals greater than or equal to $\omega_{2}$. Note that we are not assuming anything about how the $\kappa_{n}$ 's are ordered. For each $n<\omega$, we fix a $\kappa_{n}$-complete uniform ideal $I_{n}$ on $\kappa_{n}$.

Definition 6.1. Let $\mathbb{P}$ be the forcing poset whose conditions are trees of finite sequences $S$ satisfying:

(1) there is $\eta^{*} \in S$ such that for all $\nu \in S, \nu$ and $\eta^{*}$ are comparable;

(2) for all $\nu \in S$, if $\eta^{*} \unlhd \nu$ then $\nu$ is a splitting node and $\operatorname{Suc}_{S}(\nu) \in I_{\operatorname{lh}(\nu)}^{+}$

Let $T \leq S$ if $T \subseteq S$.

The node $\eta^{*}$ described in Definition 6.1 is obviously unique. We call this node the stem of $S$. Note that for all $\nu \in S$, if $\nu \triangleleft \eta^{*}$, then $\nu$ is not a splitting node.

We introduce reflexive and transitive relations $\leq^{*}$ and $\leq_{n}$, for each $n<\omega$, on $\mathbb{P}$. Define $T \leq^{*} S$ if $T \leq S$ and $S$ and $T$ have the same stem. For $n<\omega$, define $T \leq_{n} S$ if $T \leq S$ and $S$ and $T$ have the same nodes of length $n$. Note that $m \leq n$ and $T \leq_{n} S$ imply that $T \leq_{m} S$.

A sequence $\left\langle T_{n}: n<\omega\right\rangle$ is a fusion sequence if for all $n<\omega, T_{n+1} \leq_{n} T_{n}$ and $T_{n+1} \leq^{*} T_{n}$. If $\left\langle T_{n}: n<\omega\right\rangle$ is a fusion sequence, then it is easy to check that

$$
\bigcap_{n<\omega} T_{n}=\bigcup_{n<\omega}\left\{\nu \in T_{n}: \operatorname{lh}(\nu)=n\right\} .
$$

Lemma 6.2. Suppose that $\left\langle T_{n}: n<\omega\right\rangle$ is a fusion sequence. Let $T:=\bigcap\left\{T_{n}: n<\right.$ $\omega\}$. Then $T \in \mathbb{P}$, and for all $n<\omega, T \leq^{*} T_{n}$ and $T \leq_{n} T_{n}$.

Proof. Straightforward.

The next lemma describes the process which we will use for constructing fusion sequences.

Lemma 6.3. Suppose that $\left\langle T_{n}: n<\omega\right\rangle$ is a sequence of conditions, where $T_{0}$ has a stem of length $m$, satisfying:

(1) $T_{0}=T_{k}$ for all $k \leq m$;

(2) for all $n \geq m$, for all $\nu \in T_{n}$ of length $n$, there is a set $\operatorname{Suc}(\nu) \subseteq \operatorname{Suc}_{T_{n}}(\nu)$ in $I_{n}^{+}$such that

$$
T_{n+1}=\bigcup\left\{U(\nu, \xi): \nu \in T_{n}, \operatorname{lh}(\nu)=n, \xi \in \operatorname{Suc}(\nu)\right\},
$$

where $U(\nu, \xi) \leq^{*}\left(T_{n}\right)_{\nu}{ }^{-\xi}$ for all $\nu$ and $\xi$.

Then $\left\langle T_{n}: n<\omega\right\rangle$ is a fusion sequence. Moreover, letting $T:=\bigcap\left\{T_{n}: n<\omega\right\}$, we have that for all $n<\omega$, the set

$$
\left\{U(\nu, \xi): \nu \in T_{n}, \operatorname{lh}(\nu)=n, \xi \in \operatorname{Suc}(\nu)\right\}
$$

is an antichain which is predense below $T$.

\footnotetext{
${ }^{2}$ Recall that for any ideal $I$ on a set $X, I^{+}$denotes the collection of subsets of $X$ which are not in $I$.
} 
Proof. The proof is straightforward. For the second part, note that for a given $\nu$ in $T_{n}$ of length $n$ and distinct $\xi$ and $\gamma$ in $\operatorname{Suc}(\nu), U(\nu, \xi) \cap U(\nu, \gamma)$ is the set of initial segments of $\nu$. Thus, $U(\nu, \xi)$ and $U(\nu, \gamma)$ are incompatible. Suppose that $V \leq T$. Then choosing any $\nu \in V$ with $\operatorname{lh}(\nu)=n$ and $\xi \in \operatorname{Suc}_{V}(\nu)$, it is easy to see that $V_{\nu^{-} \xi}$ is below both $V$ and $U(\nu, \xi)$.

The next four results follow from standard Namba forcing type arguments; also see [10] and [11, Chapter XI].

Lemma 6.4. Let $D$ be a dense open subset of $\mathbb{P}$. Then for each $S \in \mathbb{P}$, there is $W \leq^{*} S$ and $n<\omega$ which is greater than or equal to the length of the stem of $S$ such that for any $\nu \in W$ with $\operatorname{lh}(\nu)=n, W_{\nu} \in D$.

Proof. For any condition $S$, let us say that $S$ is correct for $D$ if there exists $W \leq * S$ and $n<\omega$ which is greater than or equal to the length of the stem of $S$ such that for all $\nu \in W$ with $\ln (\nu)=n, W_{\nu} \in D$. Our goal is to show that every condition is correct for $D$. Note that if $W \leq^{*} T$ and $W \in D$, then $T$ is correct for $D$, as witnessed by the number $n$ which is the length of the stem of $T$.

Claim: If $T$ is not correct for $D$ and $\eta$ is the stem of $T$, then the set of $\gamma \in \operatorname{Suc}_{T}(\eta)$ such that $T_{\eta^{\wedge} \gamma}$ is correct for $D$ is in $I_{\operatorname{lh}(\eta)}$.

Let $A$ be the set of $\gamma \in \operatorname{Suc}_{T}(\eta)$ such that $T_{\eta^{\wedge} \gamma}$ is correct for $D$, and suppose for a contradiction that $A \notin I_{\mathrm{lh}(\eta)}$. Then $A \in I_{\mathrm{lh}(\eta)}^{+}$.

For each $\gamma \in A$, fix $U(\gamma) \leq^{*} T_{\eta^{\wedge} \gamma}$ and $n_{\gamma}<\omega$ greater than or equal to $\operatorname{lh}(\eta)+1$ such that for any $\nu$ in $U(\gamma)$ with $\operatorname{lh}(\nu)=n_{\gamma}, U(\gamma)_{\nu}$ is in $D$. Since $I_{\operatorname{lh}(\eta)}$ is $\kappa_{\operatorname{lh}(\eta)^{-}}$ complete, we can fix $n<\omega$ such that the set $A_{n}:=\left\{\gamma \in A: n_{\gamma}=n\right\}$ is in $I_{\mathrm{lh}(\eta)}^{+}$. Now define $U:=\bigcup\left\{U(\gamma): \gamma \in A_{n}\right\}$. Then $U$ is a condition and $U \leq^{*} T$.

Note that $n$ is greater than the length of the stem $\eta$ of $U$. We claim that if $\nu \in U$ and $\operatorname{lh}(\nu)=n$, then $U_{\nu} \in D$. This implies that $T$ is correct for $D$, which is a contradiction. Suppose that $\nu \in U$ and $\ln (\nu)=n$. Fix $\gamma \in A_{n}$ such that $\nu \in U(\gamma)$. Then $U(\gamma)_{\nu} \in D$. But since $n$ is greater than the length of the stem of $U, U(\gamma)_{\nu}=U_{\nu}$, so $U_{\nu} \in D$. This completes the proof of the claim.

Now let $S$ be a condition, and we will prove that $S$ is correct for $D$. Suppose for a contradiction that it is not. We define a fusion sequence $\left\langle T_{n}: n<\omega\right\rangle$. Our inductive hypothesis is that if $\nu \in T_{n}$ and $\operatorname{lh}(\nu)=n$, then $\left(T_{n}\right)_{\nu}$ is not correct for $D$. Let $\eta$ be the stem of $S$, and let $n^{*}:=\ln (\eta)$.

Define $T_{m}:=S$ for all $m \leq n^{*}$. Then for any $\nu \in T_{m}$ with $\operatorname{lh}(\nu)=m, \nu \unlhd \eta$, so $\left(T_{m}\right)_{\nu}=S$, which is not correct for $D$. Thus, the inductive hypothesis holds.

Let $m \geq n^{*}$, and assume that $T_{m}$ is defined and satisfies the inductive hypothesis. Consider any $\nu \in T_{m}$ with $\operatorname{lh}(\nu)=m$. Then by the inductive hypothesis, $\left(T_{m}\right)_{\nu}$ is not correct for $D$. Moreover, since the stem of $T_{m}$ is equal to $\eta$, which has length $n^{*}$, and $m \geq n^{*}$, it follows that $\nu$ is the stem of $\left(T_{m}\right)_{\nu}$. So by the claim, letting $B_{\nu}$ be the set of $\gamma \in \operatorname{Suc}_{T_{m}}(\nu)$ such that $\left(T_{m}\right)_{\nu^{-} \gamma}$ is not correct for $D$, we have that $\operatorname{Suc}_{T_{m}}(\nu) \backslash B_{\nu}$ is in $I_{m}$. Thus, $B_{\nu} \in I_{m}^{+}$.

Define

$$
T_{m+1}:=\bigcup\left\{\left(T_{m}\right)_{\nu ` \gamma}: \nu \in T_{m}, \operatorname{lh}(\nu)=m, \gamma \in B_{\nu}\right\}
$$


Then $T_{m+1} \leq_{m} T_{m}$. Let us verify the inductive hypothesis for $T_{m+1}$. Suppose that

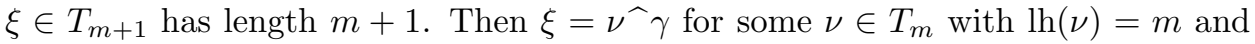
$\gamma \in B_{\nu}$. By definition, $\left(T_{m}\right)_{\nu^{\wedge} \gamma}$ is not correct for $D$, and $\left(T_{m}\right)_{\nu^{\wedge} \gamma}=\left(T_{m+1}\right)_{\xi}$.

This completes the construction of the fusion sequence $\left\langle T_{n}: n<\omega\right\rangle$. Let $T:=$ $\bigcap\left\{T_{n}: n<\omega\right\}$. Then $T \in \mathbb{P}$, and $T \leq_{n} T_{n}$ for all $n<\omega$. Since $D$ is dense, we can fix $W \leq T$ in $D$. Let $\nu$ be the stem of $W$, and fix $m$ with $\operatorname{lh}(\nu)=m$. Since the stem of $T$ is $\eta, m \geq \operatorname{lh}(\eta)=n^{*}$. Then $\nu \in T$, and hence $\nu \in T_{m}$. By the inductive hypothesis for $m$, we have that $\left(T_{m}\right)_{\nu}$ is not correct for $D$. But since $\nu$ is the stem of $W, W \leq^{*}\left(T_{m}\right)_{\nu}$. Since $W \in D$, it follows that $\left(T_{m}\right)_{\nu}$ is correct for $D$, which is a contradiction.

Lemma 6.5. Let $m<\omega$ and $\lambda$ be an ordinal, and suppose that for all $n \geq m$, $\lambda<\kappa_{n}$. Suppose that $S \in \mathbb{P}$ and the stem of $S$ has length at least $m$, and assume that $S \Vdash \dot{\alpha}<\lambda$. Then there is $T \leq{ }^{*} S$ such that $T$ decides the value of $\dot{\alpha}$.

Proof. Let $\eta$ be the stem of $S$ and let $n^{*}:=\operatorname{lh}(\eta)$. Then $m \leq n^{*}$. Let $D$ be the dense open set of conditions which are either incompatible with $S$, or below $S$ and decide the value of $\dot{\alpha}$. By Lemma 6.4 , let $k$ be the smallest natural number greater than or equal to $n^{*}$ such that there exists $T \leq^{*} S$ satisfying that for every node $\nu \in T$ with length $k, T_{\nu} \in D$. We claim that $k=n^{*}$. Then since the stem $\eta$ is a node of $T$ of length $n^{*}$, the claim implies that $T_{\eta}=T$ decides the value of $\dot{\alpha}$, and we are done.

Suppose for a contradiction that $k>n^{*}$. We will prove that there is $W \leq * S$ such that any node $\nu$ of $W$ of length $k-1$ satisfies that $W_{\nu}$ is in $D$. This will contradict the minimality of $k$ and finish the proof.

Consider any node $\nu$ of $T$ of length $k-1$. Then since $k-1 \geq n^{*}$, it follows that $\nu$ is a splitting node of $T$. By the choice of $T$ and $k$, we have that for any $\gamma \in \operatorname{Suc}_{T}(\nu)$, $T_{\nu^{-} \gamma}$ is in $D$, and hence decides $\dot{\alpha}$ to be equal to some ordinal $\alpha(\nu, \gamma)<\lambda$. There are at most $\lambda$ many possibilities for $\alpha(\nu, \gamma)$. Since $k-1 \geq n^{*} \geq m, \lambda<\kappa_{k-1}$. As $I_{k-1}$ is $\kappa_{k-1}$-complete, we can find an ordinal $\alpha(\nu)<\lambda$ and a set $\operatorname{Suc}(\nu) \subseteq \operatorname{Suc}_{T}(\nu)$ which is in $I_{k-1}^{+}$such that for all $\gamma \in \operatorname{Suc}(\nu), \alpha(\nu, \gamma)=\alpha(\nu)$.

Now define

$$
W:=\bigcup\left\{T_{\nu^{-} \gamma}: \nu \in T, \operatorname{lh}(\nu)=k-1, \gamma \in \operatorname{Suc}(\nu)\right\} .
$$

Then $W \leq^{*} T \leq^{*} S$, and so $W \leq^{*} S$. To complete the proof, we show that for any node $\nu \in W$ with length $k-1, W_{\nu} \in D$. So let $\nu \in W$ have length $k-1$. Then for any $\gamma \in \operatorname{Suc}_{W}(\nu), W_{\nu^{\wedge} \gamma}=T_{\nu^{\wedge} \gamma}$ forces that $\dot{\alpha}$ is equal to $\alpha(\nu)$. It easily follows that $W_{\nu}$ forces that $\dot{\alpha}$ is equal to $\alpha(\nu)$, and hence $W_{\nu} \in D$.

Corollary 6.6. For any $S \in \mathbb{P}$ and any statement $\varphi$ in the forcing language for $\mathbb{P}$, there is $T \leq{ }^{*} S$ which decides the truth value of $\varphi$.

Proof. Let $\dot{\alpha}$ be a $\mathbb{P}$-name which is forced to be equal to 1 if $\varphi$ is true, and 0 if $\varphi$ is false. Now apply Lemma 6.5 letting $m=0$ and $\lambda=2$.

Proposition 6.7. Let $\kappa:=\lim \sup \left\{\kappa_{n}: n<\omega\right\}$. Then for any regular cardinal $\lambda>\kappa, \mathbb{P}$ forces that $\operatorname{cf}(\lambda)>\omega$.

Proof. Let $S$ be a condition and suppose that $S$ forces that $\dot{f}: \omega \rightarrow \lambda$ is a function. We will find $T \leq S$ which forces that $\dot{f}$ is bounded in $\lambda$. It suffices to find $T \leq S$ and a set $A$ of size at most $\kappa$ such that $T$ forces that $\operatorname{ran}(\dot{f}) \subseteq A$. 
Define a fusion sequence $\left\langle T_{n}: n<\omega\right\rangle$ as follows. Fix $m<\omega$ which is greater than or equal to the length of the stem of $S \operatorname{such}$ that $\kappa=\sup \left\{\kappa_{n}: m \leq n\right\}$. Fix $\eta \in S$ with $\operatorname{lh}(\eta)=m$. For all $n \leq m$, let $T_{n}:=S_{\eta}$.

Let $n \geq m$, and assume that $T_{k}$ is defined for all $k \leq n$. We will define $T_{n+1}$. Consider a node $\nu \in T_{n}$ of length $n$. Let $D_{n}$ be the dense open set of conditions which are either incompatible with $S$, or below $S$ and decide the value of $\dot{f}(n-m)$. Applying Lemma 6.4 to $\left(T_{n}\right)_{\nu}$ and $D_{n}$, we can fix $n(\nu)<\omega$ greater than or equal to $n$ and $U(\nu) \leq^{*}\left(T_{n}\right)_{\nu}$ such that for any node $\sigma$ in $U(\nu)$ of length $n(\nu), U(\nu)_{\sigma}$ is in $D_{n}$. Let $\xi(\nu, \sigma)$ denote the ordinal such that $U(\nu)_{\sigma}$ forces that $\dot{f}(n-m)=\xi(\nu, \sigma)$. Define

$$
T_{n+1}:=\bigcup\left\{U(\nu): \nu \in T_{n}, \operatorname{lh}(\nu)=n\right\} .
$$

Then $T_{n+1} \leq_{n} T_{n}$. Let

$$
A_{n}:=\left\{\xi(\nu, \sigma): \nu \in T_{n}, \operatorname{lh}(\nu)=n, \sigma \in U(\nu), \operatorname{lh}(\sigma)=n(\nu)\right\} .
$$

It is easy to check that $T_{n+1} \Vdash \dot{f}(n-m) \in \check{A}_{n}$.

We claim that $\left|A_{n}\right| \leq \kappa$. If $\nu \in T_{n}$ and $\operatorname{lh}(\nu)=n$, then $\eta \unlhd \nu$. Since $\kappa_{k} \leq \kappa$ for all $k$ with $\operatorname{lh}(\eta)=m \leq k \leq n$, there are at most $\kappa$ many $\nu \in T_{n}$ with $\operatorname{lh}(\nu)=n$. If $\sigma \in U(\nu)$ and $\operatorname{lh}(\sigma)=n(\nu)$, then since $\kappa_{k} \leq \kappa$ for all $k$ with $n<k \leq n(\nu)$, there are at most $\kappa$ many possibilities for $\sigma$. It follows that $\left|A_{n}\right| \leq \kappa$.

This completes the construction. Let $T:=\bigcap\left\{T_{n}: n<\omega\right\}$ and $A:=\bigcup\left\{A_{n}\right.$ : $n<\omega\}$. Then $T \leq S$ and $T$ forces that $\operatorname{ran}(\dot{f}) \subseteq A$. Since $\left|A_{n}\right| \leq \kappa$ for all $n<\omega$, $|A| \leq \kappa<\lambda$.

Proposition 6.8. The forcing poset $\mathbb{P}$ forces that there exists a countable set a such that for any set $c$ in the ground model with size less than $\lim \inf \left\{\kappa_{n}: n<\omega\right\}$ in the ground model, $a \cap c$ is finite.

In particular, $\mathbb{P}$ forces that there is a countable set which is not covered by any countable set in the ground model.

Proof. Let $\dot{F}$ be a $\mathbb{P}$-name for a function such that $\mathbb{P}$ forces that for all $n<\omega$, $\dot{F}(n)=\alpha$ iff there is $S \in \dot{G}_{\mathbb{P}}$ with stem $\eta$ of length greater than $n$ such that $\eta(n)=\alpha$. It is straightforward to check that $\mathbb{P}$ forces that $\dot{F}$ is well-defined and is a total function on $\omega$. Let $\dot{a}$ be a $\mathbb{P}$-name for the range of $\dot{F}$.

Let $c$ be a set with size less than $\lim \inf \left\{\kappa_{n}: n<\omega\right\}$ and $S \in \mathbb{P}$, and we will find $T \leq S$ which forces that $\dot{a} \cap c$ is finite. Fix an integer $m$ which is greater than or equal to the length of the stem of $S$ such that for all $n \geq m,|c|<\kappa_{n}$. We define a fusion sequence $\left\langle T_{n}: n<\omega\right\rangle$. Fix $\eta \in S$ with $\operatorname{lh}(\eta)=m$, and let let $T_{n}:=S_{\eta}$ for all $n \leq m$.

Let $n \geq m$ be given, and assume that $T_{n}$ is defined. For each $\nu \in T_{n}$ of length $n$, define $\operatorname{Suc}(\nu):=\operatorname{Suc}_{T_{n}}(\nu) \backslash c$. Since $|c|<\kappa_{n}$ and $I_{n}$ is $\kappa_{n}$-complete, $\operatorname{Suc}(\nu) \in I_{n}^{+}$. Note that for all $\gamma \in \operatorname{Suc}(\nu),\left(T_{n}\right)_{\nu}{ }_{\gamma}$ forces that $\dot{F}(n)=\gamma \notin c$. Now let

$$
T_{n+1}:=\bigcup\left\{\left(T_{n}\right)_{\nu^{-} \gamma}: \nu \in T_{n}, \operatorname{lh}(\nu)=n, \gamma \in \operatorname{Suc}(\nu)\right\} .
$$

This completes the construction of $\left\langle T_{n}: n<\omega\right\rangle$. Let $T:=\bigcap\left\{T_{n}: n<\omega\right\}$. We claim that $T$ forces that $\dot{a} \cap c \subseteq \operatorname{ran}(\eta)$. First, since $\eta$ is the stem of $T, T$ forces that $\dot{F} \uparrow m=\eta$.

Secondly, consider $n \geq m$. Then by Lemma 6.3 , the set

$$
\left\{\left(T_{n}\right)_{\nu^{-} \gamma}: \nu \in T_{n}, \operatorname{lh}(\nu)=n, \gamma \in \operatorname{Suc}(\nu)\right\}
$$


is predense below $T$. But any condition in this set forces that $\dot{F}(n) \notin c$. Therefore, $T$ forces that $\dot{F}(n) \notin c$. It follows that $T$ forces that anything which is in both $\dot{a}=\operatorname{ran}(\dot{F})$ and in $c$ is in the range of $\dot{F} \uparrow m=\eta$.

For the second statement, note that $\omega<\lim \inf \left\{\kappa_{n}: n<\omega\right\}$.

Corollary 6.9. The forcing poset $\mathbb{P}$ does not have the $\omega_{1}$-approximation property.

Proof. Immediate from Proposition 6.8.

It is not necessarily the case that the Namba forcing $\mathbb{P}$ satisfies the weak $\omega_{1}$ approximation property. For example, assume $\mathrm{CH}$ and $\kappa_{n}=\omega_{2}$ for all $n<\omega$. Then by [10] and [11, Chapter XI], $\mathbb{P}$ does not add any reals. On the other hand, since $\mathbb{P}$ adds a countable cofinal subset of $\omega_{2}$, it collapses $\omega_{2}$, and therefore adds a new subset of $\omega_{1}$. But since $\mathbb{P}$ does not add reals, all of the proper initial segments of the characteristic function of the new subset of $\omega_{1}$ are in the ground model. Therefore, $\mathbb{P}$ does not have the weak $\omega_{1}$-approximation property.

In order to find a Namba forcing which does have the weak $\omega_{1}$-approximation property, we need to make an additional assumption about the ideals $I_{n}$, for $n<\omega$.

Assumption 6.10. For each $n<\omega$, there is a regular uncountable cardinal $\mu_{n} \leq$ $\kappa_{n}$ and $a$ set $P_{n} \subseteq I_{n}^{+}$satisfying:

(1) for every $A \in I_{n}^{+}$, there is $B \in P_{n}$ such that $B \subseteq A$;

(2) if $\left\langle A_{i}: i<\delta\right\rangle$ is $a \subseteq$-decreasing sequence of sets in $P_{n}$, where $\delta<\mu_{n}$, then there is $B \in P_{n}$ such that $B \subseteq \bigcap\left\{A_{i}: i<\delta\right\}$.

In the next section we will describe a model in which Assumption 6.10 holds.

Definition 6.11. Let $\mathbb{P}^{\prime}$ denote the suborder of $\mathbb{P}$ consisting of conditions $S$ satisfying that for any splitting node $\nu \in S, \operatorname{Suc}_{S}(\nu) \in P_{\operatorname{lh}(\nu)}$.

Lemma 6.12. The set $\mathbb{P}^{\prime}$ is dense in $\mathbb{P}$. In fact, for all $S \in \mathbb{P}$, there is $T \in \mathbb{P}^{\prime}$ such that $T \leq^{*} S$.

Proof. Let $m$ be the length of the stem of $S$. Define a fusion sequence $\left\langle T_{n}: n<\omega\right\rangle$ as follows. Let $T_{n}:=S$ for all $n \leq m$.

Let $n \geq m$, and assume that $T_{n}$ is defined. Let $\nu$ be a node of $T_{n}$ with length $n$. Pick a set $\operatorname{Suc}(\nu)$ in $P_{n}$ such that $\operatorname{Suc}(\nu) \subseteq \operatorname{Suc}_{T_{n}}(\nu)$. Define

$$
T_{n+1}=\bigcup\left\{\left(T_{n}\right)_{\nu^{-} \gamma}: \nu \in T_{n}, \operatorname{lh}(\nu)=n, \gamma \in \operatorname{Suc}(\nu)\right\} .
$$

Now let $T:=\bigcap\left\{T_{n}: n<\omega\right\}$. Then $T \in \mathbb{P}$, and for all $n<\omega, T \leq^{*} T_{n}$ and $T \leq_{n} T_{n}$. In particular, the stem of $T$ is equal to the stem of $S$.

To show that $T \in \mathbb{P}^{\prime}$, let $\nu \in T$ be a splitting node, and let $n$ be the length of $\nu$. Then $m \leq n$ and $\nu \in T_{n+1}$. Since $T \leq_{n+1} T_{n+1}, T$ and $T_{n+1}$ have the same nodes of length $n+1$. In particular, $\operatorname{Suc}_{T}(\nu)=\operatorname{Suc}_{T_{n+1}}(\nu)=\operatorname{Suc}(\nu) \in P_{n}$. So $T \in \mathbb{P}^{\prime}$.

Lemma 6.13. Let $m<\omega$, and suppose that $\delta$ is a limit ordinal such that $\delta<\mu_{n}$ for all $n \geq m$. Let $\left\langle T_{i}: i<\delta\right\rangle$ be $a \leq^{*}$-descending sequence of conditions in $\mathbb{P}^{\prime}$ such that the stem of $T_{0}$ has length at least $m$. Then there is $W \in \mathbb{P}^{\prime}$ such that $W \leq^{*} T_{i}$ for all $i<\delta$.

Proof. Let $T:=\bigcap\left\{T_{i}: i<\delta\right\}$. Then $T \subseteq T_{i}$ for all $i<\delta$. Since each condition $T_{i}$ has the same stem $\eta^{*}$, we also have that $\eta^{*} \in T$ and every node in $T$ is comparable with $\eta^{*}$. 
Consider a node $\nu \in T$ such that $\eta^{*} \unlhd \nu$, and we will show that $\operatorname{Suc}_{T}(\nu) \in I_{\mathrm{lh}(\nu)}^{+}$. As $\operatorname{lh}(\nu) \geq m, \delta<\mu_{\operatorname{lh}(\nu)}$. Since $\nu \in T$, for all $i<\delta, \nu \in T_{i}$. As $T_{j} \leq^{*} T_{i}$ for all $i<j<\delta$, we have that the sequence

$$
\left\langle\operatorname{Suc}_{T_{i}}(\nu): i<\delta\right\rangle
$$

is a $\subseteq$-decreasing sequence of sets in $P_{\operatorname{lh}(\nu)}$. Since $\delta<\mu_{\operatorname{lh}(\nu)}$, Assumption 6.10(2) implies that there is $A \in P_{\operatorname{lh}(\nu)}$ such that $A \subseteq \bigcap\left\{\operatorname{Suc}_{T_{i}}(\nu): i<\delta\right\}$. In particular, this intersection is in $I_{\operatorname{lh}(\nu)}^{+}$. Therefore,

$$
\operatorname{Suc}_{T}(\nu)=\bigcap\left\{\operatorname{Suc}_{T_{i}}(\nu): i<\delta\right\} \in I_{\mathrm{lh}(\nu)}^{+} .
$$

It follows that $T$ is a condition and $T \leq^{*} T_{i}$ for all $i<\delta$. Now apply Lemma 6.12 to find $W \in \mathbb{P}^{\prime}$ such that $W \leq * T$. Then $W \leq{ }^{*} T_{i}$ for all $i<\delta$.

Proposition 6.14. Let $\kappa:=\liminf \left\{\mu_{n}: n<\omega\right\}$. Then $\mathbb{P}$ does not add any bounded subsets of $\kappa$.

Proof. Let $\lambda<\kappa$ be a limit ordinal, $S \in \mathbb{P}$, and suppose that $S \Vdash \dot{a} \subseteq \lambda$. We will find a condition below $S$ which decides $\dot{a}$.

Fix $m$ such that for all $n \geq m, \lambda<\mu_{n}$, and moreover, $m$ is greater than or equal to the length of the stem of $S$. We will define a $\leq^{*}$-descending sequence $\left\langle T_{i}: i \leq \lambda\right\rangle$ of conditions in $\mathbb{P}^{\prime}$.

Fix $\eta \in S$ such that $\operatorname{lh}(\eta)=m$, and fix $T_{0} \in \mathbb{P}^{\prime}$ such that $T_{0} \leq^{*} S_{\eta}$. Note that $\eta$ is the stem of $T_{0}$.

Suppose that $\beta<\lambda$ and $\left\langle T_{i}: i \leq \beta\right\rangle$ is defined. Applying Corollary 6.6 and Lemma 6.12 , fix a condition $T_{\beta+1}$ in $\mathbb{P}^{\prime}$ such that $T_{\beta+1} \leq^{*} T_{\beta}$ and $T_{\beta+1}$ decides whether or not $\beta$ is in $\dot{a}$.

Assume that $\delta \leq \lambda$ is a limit ordinal and $\left\langle T_{i}: i<\delta\right\rangle$ is defined. Recall that for all $n \geq m, \delta \leq \lambda<\mu_{n}$, and the length of the stem of $T_{0}$ is equal to $m$. By Lemma 6.13, we can fix $T_{\delta} \in \mathbb{P}^{\prime}$ such that for all $i<\delta, T_{\delta} \leq * T_{i}$.

This completes the construction of the sequence. Let

$$
b:=\left\{\beta<\lambda: T_{\beta+1} \Vdash \beta \in \dot{a}\right\} .
$$

Then $T_{\lambda} \leq S$ and $T_{\lambda}$ forces that $\dot{a}$ is equal to $b$.

We now turn to showing that the forcing poset $\mathbb{P}$ has the weak $\mu$-approximation property, for all regular uncountable cardinals $\mu<\liminf \left\{\mu_{n}: n<\omega\right\}$. This will follow easily from the next proposition, which describes a stronger property of $\mathbb{P}$.

Proposition 6.15. Suppose that $\mu$ is a regular uncountable cardinal and $\mu<$ $\lim \inf \left\{\mu_{n}: n<\omega\right\}$. Then $\mathbb{P}$ forces that whenever $\left\langle a_{i}: i<\mu\right\rangle$ is a sequence of sets such that for all $i<\mu, a_{i} \in V$, then there is an unbounded set $X \subseteq \mu$ such that the sequence $\left\langle a_{i}: i \in X\right\rangle$ is in $V$.

Proof. Fix $m<\omega$ such that for all $n \geq m, \mu<\mu_{n}$. Suppose that $S$ forces that $\left\langle\dot{a}_{i}: i<\mu\right\rangle$ is a sequence such that for all $i<\mu, \dot{a}_{i} \in V$.

We will define $\mathrm{a} \leq^{*}$-descending sequence $\left\langle T_{i}: i \leq \mu\right\rangle$ of conditions in $\mathbb{P}^{\prime}$. Fix $\eta \in S$ which extends the stem of $S$ and has length at least $m$. Fix $T_{0} \in \mathbb{P}^{\prime}$ such that $T_{0} \leq^{*} S_{\eta}$. Then the length of the stem of $T_{0}$ is at least $m$.

Let $\beta<\mu$, and suppose that $\left\langle T_{i}: i \leq \beta\right\rangle$ is defined. Let $D_{\beta}$ be the dense open set of conditions which are either incompatible with $T_{\beta}$, or below $T_{\beta}$ and decide the value of $\dot{a}_{\beta}$. By Lemmas 6.4 and 6.12 , we can fix $T_{\beta+1} \leq^{*} T_{\beta}$ in $\mathbb{P}^{\prime}$ and $n_{\beta}<\omega$ 
which is greater than or equal to the length of the stem of $T_{\beta}$ such that for any $\nu \in T_{\beta+1}$ with $\operatorname{lh}(\nu)=n_{\beta},\left(T_{\beta+1}\right)_{\nu} \in D_{\beta}$.

Assume that $\delta \leq \mu$ is a limit ordinal and the sequence $\left\langle T_{i}: i<\delta\right\rangle$ is defined. Then for all $n \geq m, \delta \leq \mu<\mu_{n}$. Also, the length of the stem of $T_{0}$ is at least $m$. By Lemma 6.13, fix $T_{\delta} \in \mathbb{P}^{\prime}$ such that $T_{\delta} \leq^{*} T_{i}$ for all $i<\delta$.

This completes the construction of the sequence $\left\langle T_{i}: i \leq \mu\right\rangle$. Let $T:=T_{\mu}$. Then $T \leq S$.

For each $\beta<\mu, n_{\beta}<\omega$. Since $\mu$ is regular and uncountable, we can find $n<\omega$ such that the set $X:=\left\{\beta<\mu: n_{\beta}=n\right\}$ is unbounded in $\mu$.

Fix $\xi \in T$ such that $\operatorname{lh}(\xi)=n$, and let $W:=T_{\xi}$. Then $W \leq S$. We claim that $W$ forces that the sequence $\left\langle\dot{a}_{i}: i \in X\right\rangle$ is in $V$.

Consider $\beta \in X$. Since $\xi \in T, \xi \in T_{\beta+1}$ and $\operatorname{lh}(\xi)=n=n_{\beta}$. By the choice of $T_{\beta+1}$ and $n_{\beta},\left(T_{\beta+1}\right)_{\xi} \in D_{\beta}$. By the definition of $D_{\beta}$ and since $\left(T_{\beta+1}\right)_{\xi} \leq T_{\beta}$, there is a set $b_{\beta}$ such that $\left(T_{\beta+1}\right)_{\xi} \Vdash \dot{a}_{\beta}=\check{b}_{\beta}$. But $T \leq T_{\beta+1}$ implies that $W=T_{\xi} \leq$ $\left(T_{\beta+1}\right)_{\xi}$. Hence, $W \Vdash \dot{a}_{\beta}=\check{b}_{\beta}$.

It follows that $W$ forces that the sequence $\left\langle\dot{a}_{\beta}: \beta \in X\right\rangle$ is equal to the sequence $\left\langle b_{\beta}: \beta \in X\right\rangle$. Since the latter sequence is in $V$, we are done.

Corollary 6.16. Suppose that $\mu$ is a regular uncountable cardinal such that $\mu<$ $\lim \inf \left\{\mu_{n}: n<\omega\right\}$. Then $\mathbb{P}$ has the weak $\mu$-approximation property.

Proof. Suppose that $S$ forces that $\dot{f}: \mu \rightarrow O n$ is a function such that for all $\alpha<\mu, \dot{f}\lceil\alpha \in V$. Consider the sequence $\langle\dot{f}\lceil\alpha: \alpha<\mu\rangle$. Then $S$ forces that every member of this sequence is in $V$. By Proposition 6.15, there exist $T \leq S$, an unbounded set $X \subseteq \mu$, and a sequence $\left\langle g_{\alpha}: \alpha \in X\right\rangle$ such that $T$ forces that $\dot{f}\left\lceil\alpha=g_{\alpha}\right.$ for all $\alpha \in X$. In particular, for each $\alpha \in X, g_{\alpha}$ a function from $\alpha$ to $O n$, and for all $\alpha<\beta$ in $X, g_{\alpha}=g_{\beta}\left\lceil\alpha\right.$. It follows that $g:=\bigcup\left\{g_{\alpha}: \alpha \in X\right\}$ is a total function on $\mu$ and $T \Vdash \dot{f}=\check{g}$.

\section{THE MAIN THEOREM}

We are now ready to complete the main result of the paper, which is to construct a model in which there are stationarily many $N \in P_{\omega_{2}}\left(H\left(\aleph_{\omega+1}\right)\right)$ such that $N$ is indestructibly weakly guessing, has uniform cofinality $\omega_{1}$, and is not internally unbounded.

We will use the following well-known facts.

Theorem 7.1 (Larson). Martin's maximum is preserved after forcing with any $\omega_{2}$-directed closed forcing poset.

Proof. See [8, Theorem 4.3].

Theorem 7.2 (Laver). Let $\mu<\kappa$, where $\mu$ is regular uncountable and $\kappa$ is a measurable cardinal. Then $\operatorname{Col}(\mu,<\kappa)$ forces that there exists a $\kappa$-complete uniform ideal $I$ on $\kappa=\mu^{+}$and a set $P \subseteq I^{+}$satisfying:

(1) for all $A \in I^{+}$, there is $B \in P$ such that $B \subseteq A$;

(2) whenever $\left\langle B_{i}: i<\delta\right\rangle$ is $a \subseteq$-decreasing sequence of sets in $P$, where $\delta<\mu$, then $\bigcap\left\{B_{i}: i<\delta\right\} \in I^{+}$.

Proof. By [4, Theorem 7.6], there is a $\kappa$-complete uniform ideal $I$ on $\kappa=\mu^{+}$such that the forcing poset $P(\kappa) / I$ has a dense, $\mu$-closed subset. A straightforward 
argument using the $\kappa$-completeness of $I$ shows that this implies that the forcing poset $\left(I^{+}, \subseteq\right)$ has a dense, $\mu$-closed subset.

Theorem 7.3 (Hamkins). If $\dot{\mathbb{Q}}$ is an $\operatorname{Add}(\omega)$-name for an $\omega_{1}$-closed forcing poset, then $\operatorname{Add}(\omega) * \dot{\mathbb{Q}}$ has the $\omega_{1}$-approximation property.

Proof. See [6, Lemma 13].

We start with a ground model $V$ in which there is a supercompact cardinal $\kappa$ and an increasing sequence $\left\langle\kappa_{n}: n<\omega\right\rangle$ of measurable cardinals which are above $\kappa$. Let $\kappa_{-1}:=\omega_{1}$.

Let $\mathbb{M}$ be the standard forcing poset which collapses $\kappa$ to become $\omega_{2}$ and forces Martin's maximum (see [5, Section 1]). Let $G$ be a generic filter on $\mathbb{M}$. Then in $V[G], \kappa=\omega_{2}$ and Martin's maximum holds. Since $\mathbb{M}$ has size $\kappa$ in $V$, in $V[G]$ we still have that $\kappa_{n}$ is measurable for all $n<\omega$.

In $V[G]$, let $\left\langle\mathbb{P}_{n}, \dot{\mathbb{Q}}_{m}: n \leq \omega, m<\omega\right\rangle$ be the full support forcing iteration such that for all $n<\omega$,

$$
\Vdash_{\mathbb{P}_{n}} \dot{\mathbb{Q}}_{n}=\operatorname{Col}\left(\kappa_{n-1}^{+},<\kappa_{n}\right) .
$$

By standard arguments, $\mathbb{P}_{\omega}$ is $\omega_{2}$-directed closed, and for each $n<\omega, \mathbb{P}_{\omega}$ forces that $\kappa_{n}=\omega_{2 n+3}$.

Let $H$ be a $V[G]$-generic filter on $\mathbb{P}_{\omega}$. Since $\mathbb{P}_{\omega}$ is $\omega_{2}$-directed closed, Theorem 7.1 implies that Martin's maximum holds in $V[G][H]$.

Consider $n<\omega$. Then in $V[G], \mathbb{P}_{\omega}$ is forcing equivalent to a three-step forcing iteration of the form

$$
\mathbb{P}_{n} * \operatorname{Col}\left(\kappa_{n-1}^{+},<\kappa_{n}\right) * \mathbb{P}^{n},
$$

where $\left|\mathbb{P}_{n}\right|<\kappa_{n}$, and $\mathbb{P}^{n}$ is forced to be $\kappa_{n}^{+}$-closed. Let $H_{n} * H(n) * H^{n}$ be a $V[G]$-generic filter for the above forcing poset such that

$$
V[G][H]=V[G]\left[H_{n}\right][H(n)]\left[H^{n}\right] .
$$

Since $\mathbb{P}_{n}$ has size less than $\kappa_{n}$, in $V[G]\left[H_{n}\right], \kappa_{n}$ is a measurable cardinal. And $H(n)$ is a $V[G]\left[H_{n}\right]$-generic filter on $\operatorname{Col}\left(\kappa_{n-1}^{+},<\kappa_{n}\right)=\operatorname{Col}\left(\omega_{2 n+2},<\kappa_{n}\right)$. By Theorem 7.2 , in $V[G]\left[H_{n}\right][H(n)]$ there exists a $\kappa_{n}$-complete uniform ideal $I_{n}$ on $\kappa_{n}=\omega_{2 n+3}$ and a set $P_{n} \subseteq I_{n}^{+}$satisfying:

(1) for all $A \in I_{n}^{+}$, there is $B \in P_{n}$ such that $B \subseteq A$;

(2) whenever $\left\langle B_{i}: i<\delta\right\rangle$ is a $\subseteq$-decreasing sequence of sets in $P_{n}$, where $\delta<\omega_{2 n+2}$, then $\bigcap\left\{B_{i}: i<\delta\right\} \in I_{n}^{+}$.

Finally, since $\mathbb{P}^{n}$ is $\kappa_{n}^{+}$-closed in $V[G]\left[H_{n}\right][H(n)]$, it does not add any new subsets of $\kappa_{n}$, and therefore $I_{n}$ and $P_{n}$ satisfy exactly the same properties in the final model $V[G][H]=V[G]\left[H_{n}\right][H(n)]\left[H^{n}\right]$.

Let $W:=V[G][H]$. Then $W$ satisfies the following statements:

(1) Martin's maximum holds;

(2) for all $n<\omega, \kappa_{n}=\omega_{2 n+3}$;

(3) for all $n<\omega, I_{n}$ is a $\kappa_{n}$-complete uniform ideal on $\kappa_{n}$;

(4) for all $n<\omega, P_{n} \subseteq I_{n}^{+}$satisfies

(a) for all $A \in I_{n}^{+}$, there is $B \in P_{n}$ such that $B \subseteq A$;

(b) whenever $\left\langle B_{i}: i<\delta\right\rangle$ is a $\subseteq$-decreasing sequence of sets in $P_{n}$, where $\delta<\omega_{2 n+2}$, then $\bigcap\left\{B_{i}: i<\delta\right\} \in I_{n}^{+}$;

(5) $\liminf \left\{\kappa_{n}: n<\omega\right\}=\lim \sup \left\{\kappa_{n}: n<\omega\right\}=\aleph_{\omega}$. 
Working in the model $W$, we let $\mathbb{P}$ be the Namba forcing defined in Definition 6.1 using the sequence of cardinals $\left\langle\kappa_{n}: n<\omega\right\rangle=\left\langle\omega_{2 n+3}: n<\omega\right\rangle$ and the sequence $\left\langle I_{n}: n<\omega\right\rangle$ of ideals just described. By Proposition 6.7, for any regular cardinal $\lambda>\aleph_{\omega}, \mathbb{P}$ forces that $\operatorname{cf}(\lambda)>\omega$.

Observe that Assumption 6.10 is satisfied, where we let $\mu_{n}:=\omega_{2 n+2}$ for all $n<\omega$. And $\liminf \left\{\mu_{n}: n<\omega\right\}=\aleph_{\omega}$. It follows by Proposition 6.14 that $\mathbb{P}$ does not add any bounded subsets of $\aleph_{\omega}$. In particular, $\mathbb{P}$ preserves stationary subsets of $\omega_{1}$. By Corollary $6.16, \mathbb{P}$ has the weak $\omega_{n}$-approximation property, for all $1 \leq n<\omega$.

In $W^{\mathbb{P}}$ consider

$$
\mathbb{C}:=\operatorname{Add}(\omega) * \operatorname{Col}\left(\omega_{1},\left(2^{\aleph_{\omega+1}}\right)^{W}\right) .
$$

By Theorem 7.3 and Lemma $3.4, \mathbb{C}$ has the weak $\omega_{n}$-approximation property in $W^{\mathbb{P}}$, for all $1 \leq n<\omega$. It easily follows that $\mathbb{P} * \dot{\mathbb{C}}$ has the weak $\omega_{n}$-approximation property in $W$, for all $1 \leq n<\omega$. So $\mathbb{P} * \dot{\mathbb{C}}$ satisfies all of the assumptions of Proposition 5.4, where $\lambda=\aleph_{\omega+1}$ and $A=\left\{\omega_{n}: 1 \leq n<\omega\right\}$. Fix a set $w$ and a $\mathbb{P} * \dot{\mathbb{C}}$-name $\dot{\mathbb{Q}}$ which satisfy the conclusion of Proposition 5.4.

Since $\mathbb{C}$ is proper in $W^{\mathbb{P}}, \mathbb{P} * \dot{\mathbb{C}}$ preserves stationary subsets of $\omega_{1}$. As $\dot{\mathbb{Q}}$ is forced to be $\omega_{1}$-c.c., $\mathbb{P} * \dot{\mathbb{C}} * \dot{\mathbb{Q}}$ preserves stationary subsets of $\omega_{1}$.

We are ready to complete the proof. Working in $W$, fix a regular cardinal $\chi>\aleph_{\omega+1}$ such that $w$ and $\mathbb{P} * \dot{\mathbb{C}} * \dot{\mathbb{Q}}$ are in $H(\chi)$. Let $F: H\left(\aleph_{\omega+1}\right)^{<\omega} \rightarrow H\left(\aleph_{\omega+1}\right)$ be a function.

Using the fact that Martin's maximum holds in $W$, apply Theorem 5.1 to find a set $N \in P_{\omega_{2}}(H(\chi))$ such that $\omega_{1} \subseteq N, N$ is closed under $F, N \prec(H(\chi), \in$ $\left., \mathbb{P} * \dot{\mathbb{C}} * \dot{\mathbb{Q}}, \aleph_{\omega+1}, w\right)$, and there exists an $N$-generic filter $J$ on $\mathbb{P} * \dot{\mathbb{C}} * \dot{\mathbb{Q}}$. Let $M:=N \cap H\left(\aleph_{\omega+1}\right)$. Note that $M$ is closed under $F$.

By Proposition 5.4, $M$ is indestructibly weakly $\omega_{n}$-guessing, for all $1 \leq n<\omega$. Hence, $M$ is indestructibly weakly guessing. Also note that since $\mathbb{P} * \dot{\mathbb{C}} * \dot{\mathbb{Q}}$ forces that $\operatorname{cf}\left(\aleph_{\omega+1}\right)>\omega, \sup \left(M \cap \aleph_{\omega+1}\right)$ has cofinality $\omega_{1}$ by an argument similar to the proof of Lemma 5.2. By Lemma 3.2, it follows that $M$ has uniform cofinality $\omega_{1}$. By Lemma 5.2 and Proposition 6.8, there is a countable subset of $M \cap \aleph_{\omega}$ which is not covered by any countable set in $M$. Since $\aleph_{\omega} \in M$, this set is bounded below $\sup (M \cap O n) 3$

There are many possible variations of the above construction. For example, let $\kappa$ be supercompact and $\lambda>\kappa$ be measurable. Let $G$ be a generic filter on the forcing poset $\mathbb{M}$ described above, and let $H$ be a $V[G]$-generic filter on $\operatorname{Col}\left(\omega_{2},<\lambda\right)$. Then in $V[G][H]$, Martin's maximum holds and there is an $\omega_{3}$-complete uniform ideal $I$ on $\lambda=\omega_{3}$ and a set $P \subseteq I^{+}$which satisfy Assumption 6.10 for $\mu=\omega_{2}$.

Let $\mathbb{P}$ be the Namba forcing from Definition 6.1 , where we let $\kappa_{n}=\omega_{3}$ and $I_{n}=I$ for all $n<\omega$. Then $\mathbb{P}$ does not add any subsets of $\omega_{1}$, preserves $\omega_{2}$, changes the cofinality of $\omega_{3}$ to $\omega$, and preserves the uncountable cofinality of any regular cardinal greater than $\omega_{3}$. Moreover, $\mathbb{P}$ has the weak $\omega_{1}$-approximation property.

Arguing as above, in $V[G][H]$ we have that for any large enough regular cardinal $\theta$, there are stationarily many $N \in P_{\omega_{2}}(H(\theta))$ such that $\omega_{1} \subseteq N, N$ is indestructibly

\footnotetext{
${ }^{3}$ Observe that by Lemma 5.3 and Proposition $6.8, M$ is not $\omega_{1}$-guessing.
} 
weakly $\omega_{1}$-guessing, $\operatorname{cf}\left(\sup \left(N \cap \omega_{3}\right)\right)=\omega$, and for all regular uncountable cardinals $\lambda \in N \cup\{\theta\}$ different from $\omega_{3}, \operatorname{cf}(\sup (N \cap \lambda))=\omega_{1}$.

As another example, assume that $\kappa<\lambda$, where $\kappa$ is supercompact and $\lambda$ is measurable. Let $G$ be a generic filter on $\mathbb{M}$. In $V[G], \lambda$ is measurable, so we can let $I$ the dual ideal of a normal ultrafilter on $\lambda$. Let $\kappa_{n}:=\lambda$ and $I_{n}:=I$ for all $n<\omega$. Then in $V[G]$, we have that for any large enough regular cardinal $\theta$, there are stationarily many $N \in P_{\omega_{2}}(H(\theta))$ such that $\omega_{1} \subseteq N, N$ is indestructibly weakly $\mu$ guessing for all regular uncountable cardinals $\mu \in N \cap \kappa, \operatorname{cf}(\sup (N \cap \lambda))=\omega$, and for all regular uncountable cardinals $\nu \in N \cup\{\theta\}$ different from $\lambda, \operatorname{cf}(\sup (N \cap \nu))=\omega_{1}$.

We end the paper with several questions.

(1) Does the existence of stationarily many indestructibly weakly guessing models which are not internally unbounded follow from Martin's maximum alone?

(2) Does wGMP imply GMP, or wIGMP imply IGMP?

(3) Viale [13] proved that the existence of stationarily many $\omega_{1}$-guessing models which are internally unbounded implies $\mathrm{SCH}$. Does $\mathrm{SCH}$ follow from the existence of stationarily many weakly guessing models which are internally unbounded?

(4) Is it consistent that there exists a forcing poset which has the $\omega_{1}$-approximation property, but does not have the countable covering property?

(5) Is it consistent that Namba forcing on $\omega_{2}$ has the weak $\omega_{1}$-approximation property?

\section{REFERENCES}

[1] S. Cox and J. Krueger. Quotients of strongly proper forcings and guessing models. J. Symbolic Logic, 81(1):264-283, 2016.

[2] S. Cox and J. Krueger. Indestructible guessing models and the continuum. Fundamenta Mathematicae, 239:221-258, 2017.

[3] J. Cummings. Iterated forcing and elementary embeddings. In Handbook of set theory. Vols. 1, 2, 3, pages 775-883. Springer, Dordrecht, 2010.

[4] M. Foreman. Ideals and generic elementary embeddings. In Handbook of set theory. Vols. 1, 2, 3, pages 885-1147. Springer, Dordrecht, 2010.

[5] M. Foreman, M. Magidor, and S. Shelah. Martin's maximum, saturated ideals and non-regular ultrafilters I. Ann. of Math., 127(1):1-47, 1988.

[6] J. Hamkins. Extensions with the approximation and cover properties have no new large cardinals. Fund. Math., 180(3):257-277, 2003.

[7] J. Krueger. Internally club and approachable. Adv. Math., 213(2):734-740, 2007.

[8] P. Larson. Separating stationary reflection principles. J. Symbolic Logic, 65(1):247-258, 2000.

[9] W. Mitchell. On the Hamkins approximation property. Ann. Pure Appl. Logic, 144(1-3):126$129,2006$.

[10] K. Namba. Independence proof of $\left(\omega, \omega_{\alpha}\right)$-distributivity law in complete Boolean algebras. Comment. Math. Univ. St. Paul., 19:1-12, 1970.

[11] S. Shelah. Proper and Improper Forcing. Perspectives in Mathematical Logic. Springer-Verlag, Berlin, second edition, 1998.

[12] S. Todorčević. Some combinatorial properties of trees. Bull. London Math. Soc., 14(3):213$217,1982$.

[13] M. Viale. Guessing models and generalized Laver diamond. Ann. Pure Appl. Logic, 163(11):1660-1678, 2012.

[14] M. Viale and C. Weiss. On the consistency strength of the proper forcing axiom. Adv. Math., 228(5):2672-2687, 2011.

[15] C. Weiss. The combinatorial essence of supercompactness. Ann. Pure Appl. Logic, 163(11):1710-1717, 2012. 
[16] W.H. Woodin. The Axiom of Determinacy, Forcing Axioms, and the Non-Stationary Ideal. Walter de Gruyter, 1999.

Sean Cox, Department of Mathematics and Applied Mathematics, Virginia Commonwealth University, 1015 Floyd Avenue, PO Box 842014, Richmond, Virginia 23284

E-mail address: scox9@vcu.edu

John Krueger, Department of Mathematics, University of North Texas, 1155 Union Circle \#311430, Denton, TX 76203

E-mail address: jkrueger@unt.edu 